\title{
High-temperature ferromagnetism in Si:Mn alloys
}

\author{
V. N. Men'shov, ${ }^{1,2}$ V. V. Tugushev, ${ }^{2,3}$ S. Caprara, ${ }^{1,4}$ and E. V. Chulkov ${ }^{1,3}$ \\ ${ }^{1}$ Donostia International Physics Center (DIPC), P. de Manuel Lardizabal 4, E-20018 San Sebastián, Basque Country, Spain \\ ${ }^{2}$ RRC Kurchatov Institute, Kurchatov Square 1, RU-123182 Moscow, Russia \\ ${ }^{3}$ Departamento de Física de Materiales, Facultad de Ciencias Químicas, UPV/EHU and Centro Mixto CSIC-UPV/EHU, Apartado 1072, \\ E-20080 San Sebastián, Basque Country, Spain \\ ${ }^{4}$ Dipartimento di Fisica, Università di Roma "La Sapienza", Piazzale Aldo Moro 2, I-00185 Rome, Italy \\ (Received 17 September 2010; revised manuscript received 26 November 2010; published 5 January 2011)
}

\begin{abstract}
A possible mechanism for high-temperature ferromagnetic order in Si:Mn alloys is proposed. These materials, which are semiconducting or metallic, depending on the Mn content, are suggested to undergo phase separation. In the phase-separated state, again depending on the Mn content, Mn atoms can be gathered within nanometer-sized particles or micrometer-sized islands composed of the $\mathrm{MnSi}_{2-z}$ precipitate with $z \approx(0.25-0.30)$, which are embedded in the Mn-poor silicon matrix. We consider the $\mathrm{MnSi}_{2-z}$ precipitate to be the $\mathrm{MnSi}_{1.7}$ silicide host containing a certain amount of magnetic defects associated with unbound $\mathrm{Mn} 3 d$ orbitals. The $\mathrm{MnSi}_{1.7}$ silicide is considered to be a weak itinerant ferromagnet, where sizable spin fluctuations (paramagnons) exist far above its intrinsic Curie temperature, leading to a strong enhancement of the exchange coupling between the local moments of the defects. As a result, a significant enhancement of the temperature of onset of long-range order among the local moments may be achieved. We associate this temperature with the global Curie temperature of the precipitate. A phenomenological model is developed to determine the spatial structures and characteristics of ferromagnetic order for the cases of a bulk precipitate and of precipitate particles of various shapes. Moreover, allowing for the presence of strong quenched disorder in the precipitate, we describe short-range ferromagnetic order in the system. Experimental data on Si:Mn alloys are interpreted on the basis of our theoretical results.
\end{abstract}

DOI: 10.1103/PhysRevB.83.035201

PACS number(s): 75.50.Pp, 75.50.Cc, 75.75.-c

\section{INTRODUCTION}

The achievement of magnetic semiconducting materials which display both high-temperature ferromagnetism and good transport properties is of great interest for basic research. On the other hand, the ever-increasing demand of modern spintronics technologies has drawn the attention to dilute magnetic semiconductors, in order to investigate whether traditional nonmagnetic semiconductors can be manipulated by chemical substitution of transition magnetic metals to achieve control over the charge and spin densities. Dilute magnetic semiconductors that are compatible with the traditional silicon technology are particularly desirable, and Si-based magnetic alloys attracted considerable interest after the observation of ferromagnetic (FM) order with high Curie temperatures $T_{c}$ in $\mathrm{Si}: M n$ alloys of various compositions (the alternative notation $\mathrm{Si}_{1-x} \mathrm{Mn}_{x}$ is employed throughout the paper to refer to $\mathrm{Si}: \mathrm{Mn}$ alloys when the Mn content is specified). For instance, hightemperature ferromagnetism was detected in crystalline films prepared by post-thermal treatment of as-grown amorphous $\mathrm{Si}: \mathrm{Mn}$ with 5 at. $\%$ of dissolved $\mathrm{Mn}$ atoms ${ }^{1}$ and in single crystal Si wafers with $(0.1-0.8)$ at. \% of implanted $\mathrm{Mn}^{+}$ ions, ${ }^{2}$ with $T_{c} \approx 400 \mathrm{~K}$. Later on, a FM state with $T_{c} \approx 250 \mathrm{~K}$ was observed in uniformly doped $\mathrm{Si}_{1-x} \mathrm{Mn}_{x}$ films, with $x \approx$ (0.03-0.05), prepared by magnetron sputtering followed by fast annealing. ${ }^{3}$ Above room temperature ferromagnetism was also detected in $[\operatorname{Si}(20 \AA) / \mathrm{Mn}(t)]$ multilayers with nominal thickness $t=1,1.5$, and $2 \AA{ }^{4}{ }^{4}$

It is known that a single $\mathrm{Mn}$ atom prefers to enter in a tetrahedral interstitial position of the silicon crystal lattice and form a $\mathrm{Mn}^{2+}$ ion with effective magnetic moment $\approx 3 \mu_{B}$. According to Ref. 5, where ab initio calculations of the electron structure of dilute Si:Mn alloys have been performed, the mechanism for FM ordering in these systems is of the carrier mediated Ruderman-Kittel-Kasuya-Yosida (RKKY)/Zener-type. The calculations demonstrated that doped $\mathrm{Mn}$ atoms play a twofold role: (1) they promote the formation of local magnetic moments, and (2) they dope free carriers (holes), which mediate an exchange interaction between local moments. However, the theoretical predictions of Ref. 5 are only appropriate in the ideal case of homogeneous alloys. Detailed x-ray ${ }^{6-8}$ and transmission electron microscopy ${ }^{9}$ measurements have indicated that real $\mathrm{Si}: \mathrm{Mn}$ alloys are strongly inhomogeneous: the overwhelming majority of $\mathrm{Mn}$ atoms are located neither in the substitutional nor in the interstitial positions within the Si lattice, as the theory of Ref. 5 assumes, but form clusters with short-range crystalline structure, to some extent similar to strained and defected manganese silicides (with five to eight nearest-neighboring atoms). Either in the as-implanted or in the rapidly annealed samples such clusters are assembled into precipitates of various compositions. The magnetic state of a $\mathrm{Mn}$ atom in the precipitate differs substantially from the magnetic state of an isolated $\mathrm{Mn}$ impurity center in the $\mathrm{Si}$ lattice. For example, in Ref. 8, high-temperature FM order was not detected in the Mn-doped amorphous silicon films $\left(a-\mathrm{Si}_{1-x} \mathrm{Mn}_{x}\right)$, but the magnetic susceptibility obeyed the Curie-Weiss law in a wide range of $x$ (from 0.005 up to 0.175 ), although with extremely small effective moment. Such a behavior indicated that only a small fraction of doped $\mathrm{Mn}$ atoms give rise to the magnetization, whereas the majority of $\mathrm{Mn}$ atoms are magnetically inert (contrary to the general belief that a localized moment exists on $\mathrm{Mn}$ in $\mathrm{Si}$ ). The low-temperature ferromagnetism, with a saturation moment of $\approx 0.21 \mu_{B}$ per Mn atom, observed in a number of works, ${ }^{7-10}$ 
was attributed to nanometer-sized precipitates of the so-called tetragonal phase $\mathrm{MnSi}_{1.7}$. This phase is known to include several stoichiometric silicide compounds $\mathrm{Mn}_{n} \mathrm{Si}_{m}$, with $m / n \approx 1.7$ (such as $\mathrm{Mn}_{4} \mathrm{Si}_{7}, \mathrm{Mn}_{11} \mathrm{Si}_{19}, \mathrm{Mn}_{15} \mathrm{Si}_{26}, \mathrm{Mn}_{27} \mathrm{Si}_{47}$, etc.) which are, as a rule, weak itinerant ferromagnets with low Curie temperatures, $T_{c}<50 \mathrm{~K}$, and very small effective moments, $\approx 0.012 \mu_{B}$ per Mn atom. ${ }^{11,12}$ The intricate character of ferromagnetism in $\mathrm{Si}: \mathrm{Mn}$ alloys was revealed in Refs. 3 and 13, where the presence of a small amount of Mn atoms in the interstitial positions of the $\mathrm{Si}$ lattice, as well as the precipitation of Mn-rich nanometer-sized particles (henceforth, nanoparticles) in a Mn-poor silicon matrix, was detected by studying the structural, magnetic, and transport properties of the samples. The temperature-dependent magnetization was characterized by two $(\approx 50 \mathrm{~K} \text { and } \approx 250 \mathrm{~K})^{3}$ and three $[\approx 45 \mathrm{~K}, \approx(630-650) \mathrm{K} \text { and } \approx(805-825) \mathrm{K}]^{13}$ different critical temperatures. The authors explained their findings by the presence of various magnetic phases in the samples. Only the lowest critical temperature $(\approx 45 \mathrm{~K}$ or $\approx 50 \mathrm{~K})$ was confidently attributed to the FM phase of the manganese silicide precipitates. However, the key question about the origin of the high-temperature FM phases remained in abeyance.

In Ref. 14, the transport and magnetic properties of $\mathrm{Si}_{1-x} \mathrm{Mn}_{x}$ films with high Mn content, $x \approx 0.35$, produced by laser deposition, have been studied. These systems remarkably differ from those investigated in Refs. 1-3 as far as their transport properties are concerned. They exhibit a pronounced metallic-type (hole) conductivity and an anomalous Hall effect with a hysteretic behavior, in contrast to alloys with low Mn content, ${ }^{1-3}$ in which a semiconductor-type conductivity has been observed. At the same time, a fairly high Curie temperature, $T_{c} \approx 250 \mathrm{~K}$, and a magnetic moment of $\approx(0.1-$ 0.2) $\mu_{B}$ per $\mathrm{Mn}$ atom have been detected in Ref. 14, which are very similar to the magnetic characteristics reported in Refs. 1-3.

The experimental data discussed previously clearly demonstrate that in $\mathrm{Si}: \mathrm{Mn}$ alloys, in a wide range of manganese content, from 0.5 to 35 at. \%, FM order often appears at temperatures on the order of room temperature, both in semiconducting samples with low Mn content and in metallic samples with high Mn content. However, the mechanism of the phenomenon is still not understood. The authors of Ref. 15 were even compelled to suppose that the high-temperature ferromagnetism, detected in ion-implanted samples, is connected neither with the $\mathrm{Mn}$ dopant atoms nor with the $\mathrm{MnSi}_{1.7}$ intermetallic nanometer-sized phase and is rather caused by structural defects of the silicon lattice (for example, dangling bonds with uncoupled electrons), which occur in the process of preparation of the alloy. ${ }^{16}$ As a matter of fact, there exist very serious obstacles to interpreting the high-temperature FM ordering in Si:Mn alloys within the framework of the standard RKKY/Zener-type model of indirect exchange coupling between the local moments of Mn atoms diluted in the Si matrix, on account of the fact that, in Si:Mn materials, the dopant has a pronounced tendency toward the formation of silicide precipitates. As constituents of a silicide compound, the Mn atoms mainly contribute their $3 d$ orbitals to the chemical bonds with $\mathrm{Si}$ atoms. This leads to the appearance of bandlike itinerant electron states with delocalized spin density. On the other hand, the high-temperature ferromagnetism in the systems under consideration cannot be explained exclusively in terms of itinerant magnetism within the $\mathrm{MnSi}_{1.7}$ phase, since the Curie temperature and effective magnetic moment per $\mathrm{Mn}$ atom inherent to the silicide compounds are significantly smaller than those measured in the experiments. Thus, a new approach is required to describe the mechanism of exchange coupling that controls the magnetic state in these systems.

Inspired by the extraordinary physics of the Si:Mn alloys, in this work we propose a theoretical model, in the attempt to elucidate the origin of their ferromagnetism. We treat the Si:Mn alloy, which displays a significant tendency toward phase separation, as a two-component system. Namely, we suggest that the overwhelming majority of $\mathrm{Mn}$ atoms is concentrated within Mn-rich precipitate particles consisting of the $\mathrm{MnSi}_{2-z}$ phase with $z \approx(0.25-0.30),{ }^{7}$ embedded in a $\mathrm{Mn}$-poor matrix of crystalline silicon (throughout the paper, we use the notation $\mathrm{MnSi}_{2-z}$ to indicate the precipitate, as distinct from the notations $\mathrm{Si}_{1-x} \mathrm{Mn}_{x}$, used to indicate the nominally homogeneous alloy, and $\mathrm{MnSi}_{1.7}$, used to indicate the nominally homogeneous silicides $\mathrm{Mn}_{n} \mathrm{Si}_{m}$, with $m / n \approx$ 1.7). In principle, the content and size of these particles strongly depend on the nominal Mn concentration, preparation method, and thermal history of the sample. The characteristic size of the precipitate particles in relatively dilute alloys, displaying semiconducting character, has been estimated in Refs. 15 and 17 as $D_{0} \approx(5-40) \mathrm{nm}$. In more concentrated alloys, with metallic behavior, the precipitate nanoparticles percolate to form micrometer-sized islands. Such a situation seems to occur in Ref. 14.

As far as the $\mathrm{MnSi}_{2-z}$ precipitate is concerned, we assume it to consist of the $\mathrm{MnSi}_{1.7}$ silicide in which local magnetic defects are embedded. The $\mathrm{MnSi}_{1.7}$ bulk phase is assumed to be a weak itinerant ferromagnet, where strong spin fluctuations (paramagnons) exist far above its intrinsic Curie temperature. The presence of a certain amount of magnetic defects is assumed to be unavoidable within the $\mathrm{MnSi}_{1.7}$ host or at the interface between a $\mathrm{MnSi}_{1.7}$ particle and the $\mathrm{Si}$ matrix, due to the unbound $3 d$ orbitals of Mn. We suggest that the exchange coupling between the magnetic moments of the defects is mediated by spin fluctuations thermally excited in the $\mathrm{MnSi}_{1.7}$ host, resulting in a significant increase of the actual temperature of FM ordering, as compared to the intrinsic one. The possibility of such an enhancement in dilute magnetic semiconductors is mentioned in Ref. 18. To elucidate this delicate effect in our concrete situation, we developed a phenomenological approach which makes it possible to describe both long-range and short-range magnetic ordering at different length scales.

The plan of the paper is the following. In Sec. II we discuss in detail the setup of the problem, pointing out the nature and role of the $\mathrm{MnSi}_{2-z}$ precipitate, consisting of the $\mathrm{MnSi}_{1.7}$ silicide host with embedded magnetic defects. In Sec. III we present a survey of the theory for high-temperature ferromagnetism in the $\mathrm{MnSi}_{1.7}$ silicide host, initially assumed as homogeneous, and for the spin-fluctuation enhancement of the exchange coupling between magnetic defects embedded in the homogeneous $\mathrm{MnSi}_{1.7}$ silicide. Most of the theory is devoted to metallic samples (i.e., samples with percolated precipitate). 
In Sec. IV we release the assumption of homogeneity and study the effect of fluctuations of the Mn content within the silicide host. In Sec. V we consider the effect of the finite size of the precipitate particles in the semiconducting samples. The summary and concluding remarks are found in Sec. VI.

\section{SETUP OF THE PROBLEM}

In the ideal bulk $\mathrm{MnSi}_{1.7}$ silicide (which, as was already recalled in Sec. I, can exhibit several phases $\mathrm{Mn}_{n} \mathrm{Si}_{m}$, with $m / n \approx 1.7$, such as $\mathrm{Mn}_{4} \mathrm{Si}_{7}, \mathrm{Mn}_{11} \mathrm{Si}_{19}, \mathrm{Mn}_{15} \mathrm{Si}_{26}, \mathrm{Mn}_{27} \mathrm{Si}_{47}$, etc.) the $3 d$ states of $\mathrm{Mn}$ are strongly hybridized with the $4(s, p)$ states of $\mathrm{Si}$, so that the spin density on the $\mathrm{Mn}$ atom is almost completely delocalized. ${ }^{19,20}$ This material is classified as an exchange-enhanced paramagnet or a weak itinerant ferromagnet. Indeed, first-principle calculations ${ }^{19}$ showed that the different stoichiometric phases of $\mathrm{MnSi}_{1.7}$ are semiconducting, metallic, or half-metallic. For example, the spin-polarized calculations for $\mathrm{Mn}_{11} \mathrm{Si}_{19}, \mathrm{Mn}_{15} \mathrm{Si}_{26}$, and $\mathrm{Mn}_{27} \mathrm{Si}_{47}$ revealed that these phases are half-metallic, with full spin polarization of the carriers (holes) at the Fermi level. On the contrary, the ideally stoichiometric and unstressed $\mathrm{Mn}_{4} \mathrm{Si}_{7}$ silicide is shown to be a semiconductor, with indirect band gap, although small nonstoichiometry or stress lead to the closure of the gap, transforming the material into a metal.

It seems likely that the properties of the $\mathrm{MnSi}_{2-z}$ precipitate nanoparticles embedded in the silicon matrix are remarkably different from those of the ideal bulk $\mathrm{MnSi}_{1.7}$ silicide. Firstprinciple calculations ${ }^{20}$ clearly indicate that the magnetic state of the $\mathrm{MnSi}_{1.7}$ silicide is hardly affected by either the strain or the charge transfer which are certainly induced in the nanoparticle. Besides, these calculations suggest an important role of the $\mathrm{MnSi}_{1.7} / \mathrm{Si}$ interface that could be relevant for ferromagnetism in the nanometer-sized precipitate, different from that in the bulk $\mathrm{MnSi}_{1.7}$. Since the atomic density and the coordination number of $\mathrm{Mn}$ at the interface are smaller than those in the bulk, the corresponding $3 d$ orbitals are localized. The magnetic moment of the interfacial $\mathrm{Mn}$ calculated in Ref. $20, \approx 1 \mu_{B}$ per Mn atom, is considerably larger than that in bulk $\mathrm{MnSi}_{1.7}$ and is rather consistent with that of the interstitial $\mathrm{Mn}$ in $\mathrm{Si}, \approx(2-3) \mu_{B}$ per $\mathrm{Mn}$ atom, ${ }^{21}$ being nevertheless somewhat smaller than the nominal value, $\approx(4-5) \mu_{B}$ per Mn atom, embedded in the GaAs matrix. ${ }^{18}$

It is quite clear that the $\mathrm{Si}: \mathrm{Mn}$ samples display a significant structural disorder, whose detailed characteristics may depend on the way in which the samples are grown. In particular, the crystal lattice of the precipitate is far from displaying the regular periodicity of bulk $\mathrm{MnSi}_{1.7}$ where each $\mathrm{Mn}$ atom is coordinated to eight $\mathrm{Si}$ atoms. The $\mathrm{MnSi}_{2-z}$ precipitate occurs rather as a mixture of several $\mathrm{MnSi}_{1.7}$ phases, in which the coordination number of Mn fluctuates locally. According to Ref. 6, each $\mathrm{Mn}$ atom can have about five to eight nearest-neighboring $\mathrm{Si}$ atoms in the $\mathrm{Si}_{1-x} \mathrm{Mn}_{x}$ alloy. The lack of local structural order around the Mn site in the silicide precipitate leads to a (partial) localization of the wave function of $3 d$ valence electrons at this site. Thereby, the precipitate host is thought to contain angstrom-sized magnetic defects (henceforth designated by the symbol $\mathrm{Mn}_{\mathrm{d}}$ ) with a spin moment of $\approx(2-3) \mu_{B}$ per $\mathrm{Mn}$ atom and an electronic configuration similar to those of $\mathrm{Mn}_{\mathrm{T}}$ single centers or $\left(\mathrm{Mn}_{\mathrm{Si}}-\mathrm{Mn}_{\mathrm{T}}\right)$ dimer complexes, which are produced by $\mathrm{Mn}$ atoms in the tetrahedral interstitial $\left(\mathrm{Mn}_{\mathrm{T}}\right)$ and/or substitutional $\left(\mathrm{Mn}_{\mathrm{Si}}\right)$ positions in the Si lattice. ${ }^{21}$ The dimer complexes seem to be more favorable, according to numerical estimates, and are therefore more likely to occur in the systems under investigation.

As it follows from the preceding discussion, there are serious reasons to distinguish two components in the spin density of the $\mathrm{MnSi}_{2} z$ precipitate: the first, itinerant (delocalized), is inherent to the weak ferromagnet, while the second is essentially localized around the $\mathrm{Mn}_{\mathrm{d}}$ defects and/or the interfacial Mn atoms. Within this scenario, one can easily explain why the effective magnetic moment per Mn atom in $\mathrm{Si}_{1-x} \mathrm{Mn}_{x}$ alloys with a small dopant content is drastically different from that found in alloys with a Mn content large enough for phase separation to occur. First of all, let us assume that the size of precipitate particles (or islands) is so large that one can ignore the contribution of the interfacial $\mathrm{Mn}$ atoms to the magnetization of the precipitate and their influence upon the magnetic ordering in the interior of the particles (or islands). Then, supposing that the saturation magnetic moment per Mn atom consists of two components, i.e., the saturation moment of the bulk (not nanometer-sized) $\mathrm{MnSi}_{1.7}$ silicide, $\approx 0.01 \mu_{B}$ per $\mathrm{Mn}$ atom, and the saturation moment of $\mathrm{Mn}_{\mathrm{d}}$ defects, $\approx(2-3) \mu_{B}$ per $\mathrm{Mn}$ atom, we formally obtain the average saturation moment of $\approx(0.1-0.2) \mu_{B}$ per Mn atom for the two-component precipitate system $\left(\mathrm{MnSi}_{1.7}\right)_{1-y}\left(\mathrm{Mn}_{\mathrm{d}}\right)_{y}$, with $y \approx 0.04-0.08$. Following this estimate, one comes to the conclusion that only $4 \%-8 \%$ of all $\mathrm{Mn}$ atoms take part in the formation of the $\mathrm{Mn}_{\mathrm{d}}$ defects, which give the main contribution to the magnetization. Obviously, such a small concentration of defects carrying local magnetic moment is not apt to explain FM order at high temperatures in Si:Mn alloys within the framework of the RKKY/Zener model. Indeed, in this case, the average distance between magnetic moments, $a_{0}$, exceeds $1 \mathrm{~nm}$. Under the assumption that the carrier concentration is on the order of $\approx 10^{22} \mathrm{~cm}^{-3}$, typical for metallic silicides, $a_{0}$ is on the order of the period of the RKKY oscillations $2 k_{F} a_{0} \approx 5$, where $k_{F} \approx 2.5 \mathrm{~nm}^{-1}$ is the Fermi wave vector. So, the spin-glass regime should be expected for the magnetic defects diluted within the precipitate, whereas signatures of FM order are observed at high temperatures. Unfortunately, we are not aware of Mössbauer or neutron scattering measurements in Si:Mn materials and can only speculate about the spatial distribution of local magnetic moments and delocalized spin densities of carriers in these systems.

As we anticipated in Sec. I, the model we suggest postulates that a secondary phase occurs in Si:Mn dilute magnetic alloys, within the insulating $\mathrm{Si}$ matrix, in the form of nanometer sized precipitate particles. With increasing Mn concentration, the precipitate particles percolate through the $\mathrm{Si}$ matrix eventually forming a micrometer-sized cluster behaving as a macroscopic $\mathrm{MnSi}_{2-z}$ precipitate. Thereupon, we want to discuss and clarify three aspects of magnetic ordering in these complex, spatially inhomogeneous systems. First, within the continuous approximation, we discuss the mechanism for the spin-fluctuation-mediated exchange coupling among magnetic $\mathrm{Mn}_{\mathrm{d}}$ defects uniformly dissolved inside the infinite $\mathrm{MnSi}_{2-z}$ precipitate. Second, we show that the presence of short-range order can modify to a considerable extent the FM transition in 
the $\mathrm{MnSi}_{2-z}$ precipitate with strong quenched disorder. Third, we propose a model for a $\mathrm{MnSi}_{2-z}$ precipitate particle, in which the $\mathrm{MnSi}_{1.7} / \mathrm{Si}$ interface serves as a source of magnetic defects. The continuous approximation can be quite justified in the high-temperature region, where the characteristic scale of spin density variation in a weak itinerant ferromagnet (the correlation length) is small as compared with the precipitate size. It should be emphasized that our theory is not aimed at describing the magnetic behavior of an ensemble of local magnetic moments of single $\mathrm{Mn}$ atoms dispersed within the insulating $\mathrm{Si}$ matrix. Also the description of the exchange interaction between precipitate particles through the insulating matrix, which is relevant for the physics of phase separated systems, is out of the reach of our model and will be the object of future work.

\section{HIGH-TEMPERATURE FERROMAGNETISM IN THE HOMOGENEOUS PRECIPITATE WITH MAGNETIC DEFECTS}

For the sake of completeness, we recall here the main results of the theory for spin-fluctuation-mediated ferromagnetism in $\mathrm{Si}: \mathrm{Mn}$ alloys, which are relevant for the forthcoming analysis. For technical details, the reader is referred to Ref. 22. To describe FM ordering in homogeneous $\mathrm{MnSi}_{1.7}$, which is the weak itinerant FM host of $\mathrm{Mn}_{\mathrm{d}}$ magnetic defects in our model for the $\mathrm{MnSi}_{2-z}$ precipitate, we need to take into account the crucial contribution of thermal spin fluctuations beyond mean field. Within the standard Murata-Doniach approach, ${ }^{23,24}$ the reduction of the critical temperature with respect to the mean-field value, $T_{c}^{\mathrm{MF}}$, is described by a spin-fluctuation renormalization of the coefficient of the quadratic term of the Landau free energy functional of the host,

$$
F_{h}\{\boldsymbol{\varphi}\}=\int d \mathbf{r}\left[\alpha \varphi^{2}(\mathbf{r})+\beta \varphi^{4}(\mathbf{r})+\gamma\left(\frac{\partial \boldsymbol{\varphi}}{\partial \mathbf{r}}\right)^{2}\right] .
$$

Here, $\boldsymbol{\varphi}(\mathbf{r})$ is the collective magnetization of itinerant electrons dressed by spin fluctuations, $\alpha \equiv \alpha_{\mathrm{MF}}+\alpha_{\mathrm{SF}}$, where the meanfield contribution $\alpha_{\mathrm{MF}}$ is negative and almost temperature independent for $T \ll T_{c}^{\mathrm{MF}}$, and $\alpha_{\mathrm{SF}}$ is the contribution of thermal spin fluctuations, $\beta \approx W^{-3}$ and $\gamma \approx v_{F}^{2} W^{-3}, W$ and $v_{F}$ being the bandwidth and Fermi velocity of itinerant electrons, respectively. The phase transition temperature of the host, $T_{c}^{h}\left(\ll T_{c}^{\mathrm{MF}}\right)$, is defined by the condition $\alpha=0$ (i.e., $\alpha_{\mathrm{SF}}=$ $\left.\left|\alpha_{\mathrm{MF}}\right|\right)$. At $\stackrel{c}{T}>T_{c}^{h}$, when $\alpha>0$, within a self-consistent Gaussian approximation, we find

$$
\begin{aligned}
\alpha_{\mathrm{SF}} & =\frac{5 \beta T a^{3}}{4 \alpha \pi^{3}} \int_{|\mathbf{q}|<Q} \frac{d \mathbf{q}}{1+\zeta^{2} \mathbf{q}^{2}} \\
& =\frac{5 \beta T Q a^{3}}{\gamma \pi^{2}}\left[1-\frac{\arctan (\zeta Q)}{\zeta Q}\right] .
\end{aligned}
$$

Here, $\mathbf{q}$ is the wave vector, $Q$ is the cutoff, $a$ is the host lattice constant, and $\zeta \equiv \sqrt{\gamma / \alpha}$ is the correlation length. Letting $\zeta Q \rightarrow \infty$, we obtain the transition temperature

$$
T_{c}^{h}=\frac{\left|\alpha_{\mathrm{MF}}\right|}{\gamma Q^{2}} T_{0},
$$

where $T_{0} \equiv \pi^{2} \gamma^{2} Q /\left(5 \beta a^{3}\right) \propto v_{F} Q$ is a temperature scale. At $T>T_{c}^{h}, \zeta$ is significantly renormalized with respect to the mean-field value $\sqrt{\gamma /\left|\alpha_{\mathrm{MF}}\right|}$. Rewriting Eq. (2) as

$$
(\zeta Q)^{-2} T_{0}=\left[1-(\zeta Q)^{-1} \arctan (\zeta Q)\right] T-T_{c}^{h},
$$

it is evident that $\zeta Q \gg 1$ when $T_{c}^{h}<T \ll T_{0}$. In particular, for $T^{2} / T_{0} \ll T-T_{c}^{h} \ll T_{0}$, we find

$$
\zeta Q \approx \sqrt{\frac{T_{0}}{T-T_{c}^{h}}}
$$

So far, we described the $\mathrm{MnSi}_{1.7}$ silicide as a homogeneous weak itinerant ferromagnet, with intrinsic Curie temperature $T_{c}^{h}$ controlled by spin fluctuations. However, the $\mathrm{MnSi}_{2-z}$ precipitate is a more complex system, consisting of two components: the $\mathrm{MnSi}_{1.7}$ host and the $\mathrm{Mn}_{\mathrm{d}}$ magnetic defects. The latter play a relevant role in increasing the temperature for long-range FM order in the precipitate, as they become centers for the nucleation of regions with short-range FM order (which we call FM germ) within the paramagnetic (PM) host, at $T>T_{c}^{h}$. Moreover, it is possible to calculate the spin-fluctuation-mediated effective exchange coupling among magnetic defects, described as classical moments.

Indeed, at high temperature, $T>T_{c}^{h}(\alpha>0)$, Eq. (1) is regarded as an effective mean-field-like functional for the host with an order parameter $\varphi$ dressed by spin fluctuations. According to the concept of local phase transition, ${ }^{25}$ the perturbation caused by the $\mathrm{Mn}_{\mathrm{d}}$ magnetic defects inserted in the $\mathrm{MnSi}_{1.7}$ host is described by an additional local-field type term, $F_{d}\{\boldsymbol{\varphi}\}=\int d \mathbf{r} \lambda(\mathbf{r}) \cdot \boldsymbol{\varphi}(\mathbf{r})$, representing the exchange interaction between the spin density of itinerant electrons in the host and the local moments, $\lambda(\mathbf{r})$ being the corresponding exchange potential, so that the total free energy takes the form

$$
F\{\boldsymbol{\varphi}\}=F_{h}\{\varphi\}+F_{d}\{\varphi\} .
$$

Minimization with respect to $\varphi(\mathbf{r})$ yields the equation

$$
\left(\nabla^{2}-\zeta^{-2}\right) \boldsymbol{\varphi}(\mathbf{r})-\frac{2 \beta}{\gamma} \boldsymbol{\varphi}^{3}(\mathbf{r})=\frac{1}{2 \gamma} \lambda(\mathbf{r})
$$

Let us start with a single local moment at $\mathbf{r}=0$. Assuming that the range of $\lambda(\mathbf{r})$ is smaller than $\zeta$, we adopt the point defect approximation and write $\lambda(\mathbf{r})=\kappa \mathbf{S} \delta(\mathbf{r})$, where $\mathbf{S}$ is the magnetic moment at the $\mathrm{Mn}_{\mathrm{d}}$ defect, $\delta(\mathbf{r})$ is the delta function, and $\kappa$ is the exchange coupling constant. Within the model of $p$ - $d$ exchange between itinerant electrons and local moments, e.g., $\kappa \propto J_{p d} W^{-1}$, where $J_{p d}$ is the exchange matrix element. In the presence of the source term, the nonlinear term in Eq. (6) can be neglected at $|\mathbf{r}|>\varrho$, where $\varrho$ is a characteristic distance such that $(\varrho / \zeta) \exp (\varrho / \zeta) \approx|\kappa| S \sqrt{\beta / \gamma^{3}}$. Then, the solution of Eq. (6) can be written as

$$
\begin{gathered}
\varphi(\mathbf{r})=\frac{\kappa}{2 \gamma} G(\mathbf{r}, 0) \mathbf{S}, \\
G\left(\mathbf{r}, \mathbf{r}^{\prime}\right)=-\frac{\exp \left(-\left|\mathbf{r}-\mathbf{r}^{\prime}\right| / \zeta\right)}{4 \pi\left|\mathbf{r}-\mathbf{r}^{\prime}\right|}
\end{gathered}
$$

being the Green's function of the differential operator $\nabla^{2}-$ $\zeta^{-2}$. The redistribution of carrier spin density around a single local moment, Eqs. (7) and (8), describes the nucleation of a FM germ in the PM host caused by a single $\mathrm{Mn}_{\mathrm{d}}$ defect, with a characteristic radius equal to the renormalized correlation length $\zeta$. 
When the concentration $n_{m}$ of $\mathrm{Mn}_{\mathrm{d}}$ defects is finite, a composite magnetic state in the system may appear, as is described by Eqs. (5) and (6). In this case, $\lambda(\mathbf{r})=\sum_{i} \kappa \mathbf{S}_{i} \delta(\mathbf{r}-$ $\mathbf{R}_{i}$ ), where $\mathbf{R}_{i}$ and $\mathbf{S}_{i}$ are the random position and local moment of the $i$ th magnetic defect, respectively. We can exhibit the explicit solution of Eq. (6) only if the distance between nearest-neighboring defects is larger than $\varrho$. At $T>T_{c}^{h}$, the resulting magnetic state (which we call the mixed state) appears as the superposition of weakly overlapping FM germs nucleated against the background of the PM phase,

$$
\boldsymbol{\varphi}(\mathbf{r})=\boldsymbol{\varphi}_{\text {mix }}(\mathbf{r}) \equiv \frac{\kappa}{2 \gamma} \sum_{i} G\left(\mathbf{r}, \mathbf{R}_{i}\right) \mathbf{S}_{i},
$$

with $G\left(\mathbf{r}, \mathbf{r}^{\prime}\right)$ given by Eq. (8). Substituting this result into Eq. (5), within a second-order expansion in $\kappa$, omitting terms independent of the mutual orientation of the local moments, we obtain the contribution to the free energy due to the effective exchange coupling between local moments mediated by spin fluctuations in the host,

$$
F_{\mathrm{ex}}^{\mathrm{SF}}=\frac{1}{2} \sum_{i \neq j} J_{i j}^{\mathrm{SF}} \mathbf{S}_{i} \cdot \mathbf{S}_{j},
$$

with a nonoscillating exchange integral (see Fig. 1),

$$
J_{i j}^{\mathrm{SF}} \equiv \frac{\kappa^{2}}{\gamma} G\left(\mathbf{R}_{i}, \mathbf{R}_{j}\right)<0 .
$$

Thus, at low $n_{m}$, when the average distance among defects $a_{0} \approx n_{m}^{-1 / 3}>\varrho$, the coupling (11) favors the FM ordering of local moments. This is likely to occur also at highdefect concentration, when $a_{0} \lesssim \varrho$ (i.e., $a_{0} \ll \zeta$ ), although the calculation of $J_{i j}^{\mathrm{SF}}$ is no longer viable, since the linearization of Eq. (6) and the second-order expansion in $\kappa$ are no longer justified. Within the scenario discussed previously, hightemperature FM ordering in $\mathrm{MnSi}_{2-z}$ precipitates is described

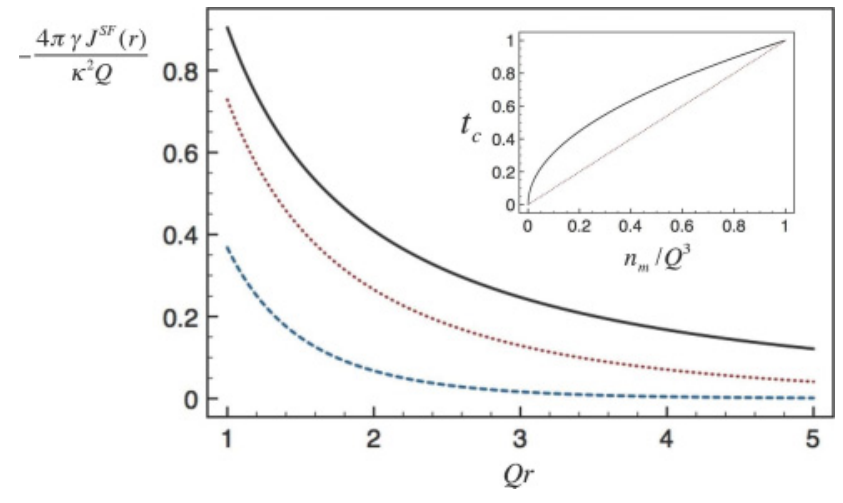

FIG. 1. (Color online) Main panel: Spin-fluctuation-mediated exchange interaction $J_{i j}^{\mathrm{SF}} \equiv J^{\mathrm{SF}}(r)$ as a function of the distance between magnetic defects $r \equiv\left|\mathbf{R}_{i}-\mathbf{R}_{j}\right|$. The coupling is made dimensionless and positive by the prefactor $-4 \pi \gamma /\left(\kappa^{2} Q\right)$. The distance is made dimensionless by the prefactor $Q$. The curves correspond, from top to bottom, to the temperatures $T-T_{c}^{h}=0.01 T_{0}$ (solid line), $T-T_{c}^{h}=$ $0.1 T_{0}$ (dotted line), and $T-T_{c}^{h}=T_{0}$ (dashed line). Inset: Critical temperature as a function of the concentration of magnetic defects $n_{m}$. The dimensionless quantity $t_{c} \equiv \sqrt{3 \gamma k_{B} /\left(\kappa^{2} S^{2} Q T_{0}\right)} T_{c}^{g}$ (solid line) is plotted as a function of the dimensionless parameter $n_{m} / Q^{3}$. For comparison, the dimensionless quantity $3 W k_{B} T_{c}^{R K K Y} /\left(J_{p d}^{2} S^{2} Q^{3}\right)$, which depends linearly on $n_{m}$, is also plotted (dotted line). as a phase transition in the two-component system at the global critical temperature $T_{c}^{g}$, below which $\left\langle\boldsymbol{\varphi}_{\text {mix }}(\mathbf{r})\right\rangle_{C} \neq 0$ and $\left\langle\sum_{i} \mathbf{S}_{i}\right\rangle_{C} \neq 0$. Here, the symbol $\langle\cdots\rangle_{C}$ implies an average over orientations and positions of the local moments. It is clear that $\left\langle\varphi_{\text {mix }}^{2}\right\rangle_{C} \neq 0$, while $\left\langle\varphi_{\text {mix }}\right\rangle_{C}=0$ at $T \geqslant T_{c}^{g}$. The overlap of the neighboring FM germs grows continuously with decreasing $T$ and, correspondingly, the exchange integral (11) increases until long-range FM order appears at $T=T_{c}^{g}$. We point out that, whereas $T_{c}^{h}$ is reckoned as the intrinsic temperature of freezing of thermal spin fluctuations in the host, $T_{c}^{g}$ is the transition temperature from the uncorrelated state of local moments to the partially aligned state, with decreasing $T$.

Within a mean-field approximation for the model in Eqs. (10) and (11), we find

$$
T_{c}^{g}=\frac{S^{2}}{3 k_{B}} J_{0}^{\mathrm{SF}}\left(T_{c}^{g}\right),
$$

where $J_{0}^{\mathrm{SF}} \equiv\left|\sum_{j} J_{i j}^{\mathrm{SF}}\right| \approx n_{m} \kappa^{2} \zeta^{2} / \gamma$ depends on $T$ via the renormalized correlation length $\zeta$. If $T_{c}^{g} \gg T_{c}^{h}$, from Eq. (4), we estimate $\zeta\left(T_{c}^{g}\right) Q \approx \sqrt{T_{0} / T_{c}^{g}}$ and hence

$$
T_{c}^{g}=\sqrt{\frac{\kappa^{2} S^{2} n_{m} T_{0}}{3 \gamma Q^{2} k_{B}}} \approx|\kappa| S \sqrt{\frac{n_{m} v_{F}}{\gamma Q k_{B}}} .
$$

Noteworthy, $T_{c}^{g}$ depends on the density of magnetic defects as $\sqrt{n_{m}}$ (see the inset in Fig. 1).

In metallic $\mathrm{MnSi}_{2-z}$ precipitates, the spin-fluctuationmediated exchange between local moments competes with the RKKY carrier-mediated exchange and a comparative estimate is necessary. A sound set of parameters for the systems under investigation is $a_{0} \approx(10-12) \AA, k_{F}^{-1} \approx(3-4) \AA$, $W \approx 10^{4} \mathrm{~K}$, and $T_{0} \approx\left(10^{3}-10^{4}\right) \mathrm{K}$. Then, the condition $\zeta \sim$ $a_{0} \gg k_{F}^{-1}$ is met if $\zeta\left(T_{c}^{g}\right)>k_{F}^{-1} \sqrt{W / T_{c}^{g}} \approx(3-4) k_{F}^{-1}$. In this regime, $J_{0}^{\mathrm{SF}} \approx n_{m} J_{p d}^{2} /\left(2 W^{2} \alpha\right)$ and $J_{0}^{\mathrm{RKKY}} / J_{0}^{\mathrm{SF}} \approx 2 W \alpha \ll 1$. Similarly, from Eq. (13), we obtain $T_{c}^{g} \approx W \sqrt{T_{c}^{\mathrm{RKKY}} / T_{0}} \gg$ $\left(T_{c}^{\mathrm{RKKY}}, T_{c}^{h}\right)$, where $T_{c}^{\mathrm{RKKY}} \approx n_{m} J_{p d}^{2} S^{2} /\left(3 W k_{B}\right)$. Even the cautious estimate $T_{c}^{\mathrm{RKKY}} \approx 10 \mathrm{~K}$ yields the rather high critical temperature $T_{c}^{g} \approx(300-500) \mathrm{K}$. In passing, we note that the critical temperature for the RKKY mechanism not only has a smaller dimensional prefactor, as compared with the critical temperature for the spin-fluctuation-mediated mechanism, but also depends linearly on $n_{m}$ (see the inset in Fig. 1).

At very low $n_{m}$, when $a_{0} \gg\left(\zeta, k_{F}^{-1}\right)$, the oscillating RKKY interaction dominates and a spin-glass state in the precipitate may become favorable. This issue, however, is outside the reach of the present study.

\section{EFFECT OF QUENCHED DISORDER ON THE FM TRANSITION IN THE PRECIPITATE}

The disorder in the positions of the $\mathrm{Mn}_{\mathrm{d}}$ centers, always present in the $\mathrm{MnSi}_{2-z}$ precipitate, leads to a spatially inhomogeneous distribution of the charge and spin density of carriers throughout the host and therefore results in a nonuniform magnetization. The aforementioned mean-field approach, of course, is not apt to capture such a tiny effect. Here, we address this issue in the case of relatively high concentration of $\mathrm{Mn}_{\mathrm{d}}$ defects, when $a_{0} \ll \zeta$. Under this circumstance, we can 
initially accomplish the averaging over the distribution of magnetic defects in the two-component alloy $\left(\mathrm{MnSi}_{1.7}\right)_{1-y}\left(\mathrm{Mn}_{\mathrm{d}}\right)_{y}$ $\left(y \propto n_{m}\right)$ over distances on the order of $a_{0}$ and imply that the coefficients in the free energy functional $F_{h}\{\varphi\} \rightarrow F_{h, r}\{\varphi\}$ are correspondingly renormalized (henceforth, the subscript $r$ indicates the renormalized coefficients). Moreover, as will become clearer later, it may be necessary to include in the functional (1) the term $\theta \varphi^{6}$. The term associated with defects, Eq. (5), is rewritten as

$$
F_{d}\{\boldsymbol{\varphi}, \Delta\}=\kappa \int d \mathbf{r}\left[n_{m}+\Delta(\mathbf{r})\right]\langle\mathbf{S}(\mathbf{r})\rangle \cdot \boldsymbol{\varphi}(\mathbf{r}),
$$

where $\Delta(\mathbf{r})$ is the random slowly varying component of the defect concentration, which is such that $\int d \mathbf{r} \Delta(\mathbf{r})=0$, when integrated over the volume of the sample. Equation (14) means that the spin density of the carriers interacts with the average magnetization of the local moments. In turn, we assume that the local moments are affected by the field of spin fluctuations,

$$
\langle S(\mathbf{r})\rangle=-S \mathcal{L}\left(\frac{\kappa S \varphi(\mathbf{r})}{k_{B} T}\right),
$$

where $\mathcal{L}(x)$ is the Langevin function. The minus sign on the right-hand side of Eq. (15) indicates that the vectors $\varphi$ and $\langle\mathbf{S}\rangle$ are parallel (antiparallel) to each other for $\kappa<0(\kappa>0)$. Inserting expression (15) for $\langle S(\mathbf{r})\rangle$ into Eq. (14), we include terms up to the fourth power in the order parameter $\varphi$ in $F_{d}$. This is quite justified at sufficiently high temperature, where $|\kappa S \varphi| /\left(k_{B} T\right) \ll 1$.

Now, the quenched disorder in the defect distribution may be referred to as a disorder of the local transition temperature type. ${ }^{26}$ In this representation, the character of the spatial distribution of defects is encoded in the statistical properties of the random field $\Delta(\mathbf{r})$. Let us assume a Gaussian density distribution,

$$
P\{\Delta\}=\exp \left[-\frac{1}{2 \omega} \int d \mathbf{r} \Delta^{2}(\mathbf{r})\right],
$$

with a single parameter, $\omega$, characterizing the strength of disorder.

To obtain the free energy for our system we have to calculate the contributions of different configurations of $\varphi(\mathbf{r})$ and $\Delta(\mathbf{r})$. This task would be awkward without some additional assumptions on the relevant configurations. Thus, we suggest that an important role is played by fluctuations in the distribution of magnetic defects that tend to form rare regions with locally enhanced or reduced density of defects, with $\Delta(\mathbf{r})>0$ and $\Delta(\mathbf{r})<0$, respectively, accompanied by a corresponding deformation of the spin density profile $\varphi(\mathbf{r})$. These regions will be henceforth called droplets. Following Ref. 26, we write the average excess free energy of the system (with respect to a homogeneous system, in the absence of disorder) as a functional integral over the variable $\Delta(\mathbf{r})$,

$$
\mathbb{F}=-k_{B} T \int \mathcal{D}\{\Delta\} \exp \left(-\frac{\mathcal{F}\{\varphi, \Delta\}}{k_{B} T}\right),
$$

up to a normalization factor. Here, the functional

$$
\mathcal{F}\{\varphi, \Delta\}=\mathcal{E}\{\varphi, \Delta\}-k_{B} T \ln P\{\Delta\}
$$

has the meaning of the free energy of the droplet with collective magnetization $\varphi(\mathbf{r})$ induced by the disorder fluctuation $\Delta(\mathbf{r})$, and

$$
\mathcal{E}\{\varphi, \Delta\} \equiv F_{h, r}\{\varphi\}+F_{d}\{\varphi, \Delta\}
$$

is the renormalized Ginzburg-Landau functional in the presence of defects, which minimizes the free energy for a given realization of disorder.

According to Ref. 26, the main contribution to the functional integral over $\Delta(\mathbf{r})$ in Eq. (16) comes from the configuration that realizes an extremum of the functional $\mathcal{F}\{\varphi, \Delta\}$. Varying the functional (17) under the constraint condition $\int d \mathbf{r} \Delta(\mathbf{r})=0$, we obtain the optimal fluctuation for the distribution of the defects:

$$
\Delta^{(\mathrm{of})}(\mathbf{r})=\frac{\omega}{3}\left(\frac{\kappa S}{k_{B} T}\right)^{2}\left[\varphi^{2}(\mathbf{r})-\overline{\varphi^{2}}\right],
$$

where $\overline{\varphi^{2}}=V^{-1} \int \varphi^{2}(\mathbf{r}) d \mathbf{r}$ is the mean square value of the ordered parameter and $V$ is the volume. Note that $\overline{\varphi^{2}}$ may be finite even if the mean value of the order parameter $\bar{\varphi}=$ $V^{-1} \int \varphi(\mathbf{r}) d \mathbf{r}$ vanishes. Here, we consider configurations for which $\overline{\varphi^{2}}-\bar{\varphi}^{2}$ vanishes in the thermodynamic limit $V \rightarrow \infty$. Thus, long-wavelength incommensurate configurations are excluded from our analysis. Of course, for spatially homogeneous configurations the equality $\overline{\varphi^{2}}=\bar{\varphi}^{2}$ holds. Our description does not allow one to calculate the density of droplets, which must be taken as a parameter, or must be calculated by means of the replica method, ${ }^{26}$ if one wishes to calculate the full contribution to the free energy.

Minimizing $\mathcal{E}\{\varphi, \Delta\}$, Eq. (18), with respect to $\varphi(\mathbf{r})$ and then substituting the expression for $\Delta^{(\text {of })}(\mathbf{r})$, Eq. (19), into the equation that corresponds to the extremal of the energy $\mathcal{E}\{\varphi, \Delta\}$, one arrives at the self-consistent equation for the spin density:

$$
\gamma \nabla^{2} \varphi-\alpha_{\omega} \varphi-2 \beta_{\omega} \varphi^{3}-3 \theta \varphi^{5}=0
$$

where

$$
\begin{gathered}
\alpha_{\omega} \equiv \alpha_{r}+2 \Omega \bar{\varphi}^{2}, \quad \alpha_{r} \equiv \alpha-\frac{(\kappa S)^{2} n_{m}}{3 k_{B} T}, \\
\beta_{\omega} \equiv \beta_{r}-\Omega, \quad \beta_{r} \equiv \beta+\frac{(\kappa S)^{4} n_{m}}{45\left(k_{B} T\right)^{3}} \\
\Omega \equiv \frac{\omega}{18} \frac{(\kappa S)^{4}}{\left(k_{B} T\right)^{3}} .
\end{gathered}
$$

In principle, also the coefficient $\theta$ is renormalized by the presence of defects and corrected by disorder. The correction due to defects is negative, so we assume that the bare coefficient is large enough to remain positive after renormalization. The correction due to quenched disorder may be shown to be positive, and therefore we take $\theta$ as a given positive constant.

If the magnitude of the concentration fluctuations is small enough, so that $\beta_{\omega}>0$, the uniform ordered phase appears below the temperature $T_{c}^{g}$ at which $\alpha_{r}=0$. The expression of the critical temperature $T_{c}^{g}$ obviously coincides with Eq. (13), since at $\omega=0$ our procedure is just a reformulation of the 
theory exposed in Sec. III. The ordered phase, according to Eq. (20), is characterized by a uniform spin density,

$$
\bar{\varphi}=\sqrt{\frac{\gamma Q^{2}}{\beta_{r}\left(T=T_{c}^{g}\right)} \frac{T_{c}^{g}-T}{T_{0}}} .
$$

Here, the term $\varphi^{5}$ is neglected. The uniform ordering of the carrier spin density is associated with the uniform FM state of the local moments with average value $\overline{\langle S\rangle}=-\kappa S^{2} \bar{\varphi} /\left(3 k_{B} T_{c}^{g}\right)$.

If the magnitude of the concentration disorder in the $\left(\mathrm{MnSi}_{1.7}\right)_{1-y}\left(\mathrm{Mn}_{\mathrm{d}}\right)_{y}$ alloy is so large that $\beta_{\omega}<0$, and therefore the term $\varphi^{6}$ must be introduced in the functional to make the system stable against fluctuations of the order parameter, the FM phase turns out to be very unusual, with a highly nonuniform magnetization. In order to illustrate this effect, we restrict ourselves to the one-dimensional geometry, which does not qualitatively change the main results of our analysis.

Let us assume that the concentration of magnetic defects is locally increased near the origin $x=0$. Above the temperature $T_{c}^{g}$, such an inhomogeneity can induce the nucleation of a state with short-range order. To determine the static profile of the spin density in this state, we search the solution of Eq. (20) which satisfies the boundary conditions $\varphi(x) \rightarrow 0$ and $(d \varphi / d x) \rightarrow 0$ when $|x| \rightarrow \infty$. A nontrivial solution exists, provided $\beta_{\omega}<0$. In the one-dimensional case, one can readily construct the first integral and give the solution of the nonlinear equation (20) in the explicit form

$$
\varphi_{d}(x)=\frac{\Gamma_{+} \Gamma_{-}}{\sqrt{\left(\Gamma_{+}^{2}-\Gamma_{-}^{2}\right) \cosh ^{2}\left(x / \zeta_{d}\right)+\Gamma_{-}^{2}}},
$$

where

$$
\Gamma_{ \pm}^{2}=\frac{\left|\beta_{\omega}\right| \pm \sqrt{\beta_{\omega}^{2}-4 \alpha_{r} \theta}}{2 \theta}
$$

and

$$
\zeta_{d} \equiv \sqrt{\frac{\gamma}{\theta}}\left(\Gamma_{+} \Gamma_{-}\right)^{-1}=\sqrt{\frac{\gamma}{\alpha_{r}}}=\frac{1}{Q} \sqrt{\frac{T_{0}}{2\left(T-T_{c}^{g}\right)}}
$$

is the correlation length of spin fluctuations in the presence of magnetic defects. One can see that FM droplets with magnetization profile $\langle S(x)\rangle \propto \varphi_{d}(x)$, Eq. (24), arise due to the presence of rare spatial regions strongly enriched in $\mathrm{Mn}_{\mathrm{d}}$, while the bulk silicide stays PM, with $\bar{\varphi}=0$. The nucleation of the locally ordered phase corresponds to the optimal concentration fluctuation $\Delta_{d}^{(\text {of })}(x) \propto \varphi_{d}^{2}(x)$ [Eqs. (19) and (24)]. If the temperature is such that $\beta_{\omega}^{2} \gtrsim 4 \alpha_{r} \theta$, the shape of such a fluctuation is characterized by a broad core, in the region $4|x| \lesssim-\zeta_{d} \ln \left(1-4 \alpha_{r} \theta / \beta_{\omega}^{2}\right)$, where the spin density is $\varphi_{d}(x) \simeq \varphi_{d}(0)=\Gamma_{-}$, while in the asymptotic region, outside the core, the spin density $\varphi_{d}(x) \approx \exp \left(-|x| / \zeta_{d}\right)$ falls off exponentially. Near the temperature $T_{c}^{g}, \beta_{\omega}^{2} \gg 4 \alpha_{r} \theta$, and one has $\varphi_{d}(x) \approx \sqrt{\alpha_{r} /\left|\beta_{\omega}\right|}\left[\cosh \left(x / \zeta_{d}\right)\right]^{-1}$.

It is clear that, in order to create the locally ordered state at a given temperature, $T>T_{c}^{g}$, the strength of the disorder must be large enough, $\beta_{\omega}^{2}>4 \alpha_{r} \theta$. On the other hand, this inequality fixes the critical temperature

$$
T_{\max }=T_{c}^{g}+\frac{T_{0} \beta_{\omega}^{2}}{8 \gamma \theta Q^{2}},
$$

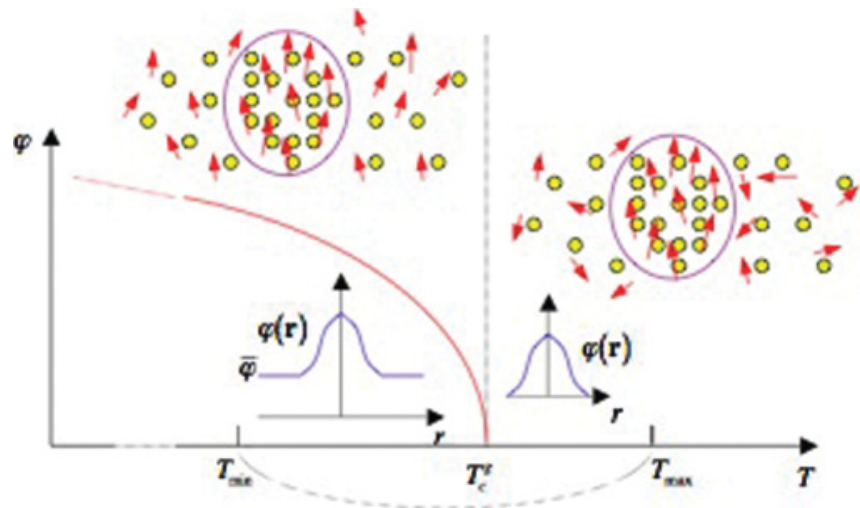

FIG. 2. (Color online) Effect of quenched disordered on the magnetic state of Si:Mn alloys around the transition point $T_{c}^{g}$ in a region strongly enriched in $\mathrm{Mn}_{d}$ defects. Within the temperature interval $T_{c}^{g}<T<T_{\max }$, FM droplets with profile $\varphi_{d}$ arise, while the bulk precipitate is in the PM phase. Within the temperature interval $T_{\min }<T<T_{c}^{g}$, the FM order is locally amplified against the background, $\varphi>\bar{\varphi}$. A pictorial view of a region enriched in $\mathrm{Mn}_{d}$ magnetic defects and of the distribution of local magnetic moments around it, in the two temperature intervals, is shown. The small shaded circles represent the $\mathrm{Mn}_{d}$ magnetic defects and the arrows represent their magnetic moment. The profile of the carrier spin density $\varphi(\mathbf{r})$ is also qualitatively sketched.

above which (within our model) quenched disorder cannot induce a locally ordered state (see Fig. 2). Note that, in Eq. (25), the parameter $\beta$ is implied to be weakly temperature dependent. As $T$ is lowered, droplets with finite amplitude $\varphi_{d}(0)=\sqrt{\left|\beta_{\omega}\right| / 2 \theta}$ appear sharply at $T_{\max }$.

We now turn to the question of how the strong quenched disorder, when $\beta_{\omega}<0$, influences the FM state below the transition temperature, at $\alpha_{r}<0$. The defect concentration is assumed to be either locally lowered or locally raised near the origin $x=0$. Correspondingly, we are interested in one of the solutions of Eq. (20), either $\varphi_{+}(x)$ or $\varphi_{-}(x)$, which meet the boundary conditions $\varphi_{ \pm}(x) \rightarrow \Theta_{ \pm}$and $\left(d \varphi_{ \pm} / d x\right) \equiv$ $\varphi_{ \pm}^{\prime}(x) \rightarrow 0$ as $|x| \rightarrow \infty$, besides $\varphi_{+}^{\prime \prime}(0)>0$ or $\varphi_{-}^{\prime \prime}(0)<0$. Under Eq. (19), the optimal fluctuations are opposite in sign, $\Delta_{+}^{(\text {of })}(x)<0$ while $\Delta_{-}^{(\text {of })}(x)>0$, and vanish asymptotically, $\Delta_{ \pm}^{\text {(of) }}(x) \rightarrow 0$ as $|x| \rightarrow \infty$. The corresponding solutions exist and may be expressed within the one-dimensional model in the explicit form

$$
\varphi_{ \pm}(x)=\frac{\Phi_{ \pm} \Theta_{ \pm} \cosh \left(x / \zeta_{ \pm}\right)}{\sqrt{\Phi_{ \pm}^{2} \sinh ^{2}\left(x / \zeta_{ \pm}\right)+\Theta_{ \pm}^{2}}},
$$

where

$$
\begin{aligned}
\Theta_{ \pm}^{2} & =\frac{\left|\beta_{\omega}\right| \pm \sqrt{\beta_{\omega}^{2}-3 \alpha_{\omega} \theta}}{3 \theta} \\
\zeta_{ \pm}^{-1} & \equiv \Theta_{ \pm} \sqrt{( \pm) \frac{\theta}{\gamma}\left(\Theta_{ \pm}^{2}-\Phi_{ \pm}^{2}\right)}
\end{aligned}
$$

and $\Phi_{ \pm}^{2}=\left(\left|\beta_{\omega}\right| / \theta\right)-2 \Theta_{ \pm}^{2}$.

According to the relations (21) and (22), the parameters of the solution (26), $\Theta_{ \pm}$and $\Phi_{ \pm}$, may be expressed through the 
coefficients $\alpha_{\omega}$ and $\beta_{\omega}$. Using Eqs. (21) and (22), we find out that $\Theta_{+}^{2}=\Theta_{-}^{2}=\bar{\varphi}^{2}$, where

$$
\bar{\varphi}^{2}=\frac{\sqrt{\beta_{r}^{2}+3\left|\alpha_{r}\right| \theta}-\beta_{r}}{3 \theta} .
$$

Evidently, the value $\bar{\varphi}$ (28) reduces to Eq. (23) at $\beta_{r}^{2} \gg 3\left|\alpha_{r}\right| \theta$. In the same manner, we obtain the value of the order parameter at the origin, $\varphi_{ \pm}(x=0)=\Phi_{ \pm}=\Phi$, where

$$
\Phi^{2}=\bar{\varphi}^{2}+\frac{\Omega-\sqrt{\beta_{r}^{2}+3\left|\alpha_{r}\right| \theta}}{\theta} .
$$

The state with the profile $\varphi_{-}(x)$ exists under the condition $\left|\alpha_{r}\right| \theta<\left(\Omega^{2}-\beta_{r}^{2}\right) / 3$, while the region of existence for the state with the profile $\varphi_{+}(x)$ is defined by the inequalities $\left(\Omega^{2}-\right.$ $\left.\beta_{r}^{2}\right) / 3<\left|\alpha_{r}\right| \theta<\left(\Omega-\beta_{r}\right)\left(3 \Omega+\beta_{r}\right) / 4$. We denote by $T_{\min }$ the temperature that fulfills the equation $\left|\alpha_{r}\right| \theta=\left(\Omega^{2}-\beta_{r}^{2}\right) / 3$. At $T=T_{\min }$ one has $\varphi_{+}(x)=\varphi_{-}(x)=\bar{\varphi}$. The redistribution of the spin density in the form $\varphi_{+}(x)\left[\varphi_{-}(x)\right]$ corresponds to the situation when an attenuation (amplification) of the FM ordering occurs in the $\mathrm{Mn}_{d}$-depleted (-rich) region, respectively, while the $\left(\mathrm{MnSi}_{1.7}\right)_{1-y}\left(\mathrm{Mn}_{\mathrm{d}}\right)_{y}$ silicide alloy is globally FM. The variation of the $\mathrm{Mn}_{\mathrm{d}}$ concentration in this region reaches the value $\Delta^{(\mathrm{of})}(0)$, which is proportional to $\Phi^{2}$, Eq. (28), its size being on the order of $\zeta_{1,2}$, Eq. (27).

In the region $\left|\alpha_{r}\right| \theta>\left(\Omega-\beta_{r}\right)\left(3 \Omega+\beta_{r}\right) / 4$, a state with a spin density profile corresponding to a domain wall occurs. The solution of Eq. (20), with the particular boundary conditions $\varphi(x) \rightarrow \pm \bar{\varphi}$ as $x \rightarrow \pm \infty$ and $\varphi(0+)=-\varphi(0-)$, can be found out via the first integral to give

$$
\varphi_{k}(x)=\frac{\Phi_{0} \bar{\varphi} \operatorname{sign}(x)}{\sqrt{\left(\bar{\varphi}^{2}+\Phi_{0}^{2}\right) \operatorname{coth}^{2}\left(x / \zeta_{0}\right)-\bar{\varphi}^{2}}},
$$

where

$$
\zeta_{0}^{-1}=2 \bar{\varphi} \Phi_{0} \sqrt{\theta / \gamma}
$$

and $\Phi_{0}^{2}=2 \bar{\varphi}^{2}-\left(\left|\beta_{\omega}\right| / \theta\right)$. We denote by $T_{\mathrm{dw}}$ the temperature that fulfills the equation $\left|\alpha_{r}\right| \theta=\left(\Omega-\beta_{r}\right)\left(3 \Omega+\beta_{r}\right) / 4$. The optimal fluctuation, which corresponds to the spin density $\varphi_{k}(x)$, Eq. (30), is given by

$$
\Delta_{k}^{(\mathrm{of})}(x)=-\frac{\omega}{3}\left(\frac{\kappa S}{k_{B} T}\right)^{2} \frac{\bar{\varphi}^{2}\left(\bar{\varphi}^{2}+\Phi_{0}^{2}\right)}{\bar{\varphi}^{2}+\Phi_{0}^{2} \cosh ^{2}\left(x / \zeta_{0}\right)} .
$$

The magnetic phase diagram of the $\mathrm{MnSi}_{2-z}$ precipitate with strong quenched disorder may be described as follows. Fluctuations in the content of $\mathrm{Mn}_{\mathrm{d}}$ defects with locally raised concentration, where $\Delta^{(\text {of })}(x)>0$, can manifest themselves within a rather wide temperature interval, $T_{\min }<T<T_{\max }$, around the Curie point of the uniform phase, $T=T_{c}^{g}$ (see Fig. 2). These fluctuations play the role of seeds for the nucleation of droplets above the temperature $T_{c}^{g}$ or deformations of the uniform FM background below the temperature $T_{c}^{g}$. It is interesting to note that optimal fluctuations in the content of $\mathrm{Mn}_{\mathrm{d}}$ defects with locally lowered concentration $\left[\Delta^{\text {(of })}(x)<0\right.$ ] do not occur within the interval $T_{\min }<T<T_{\max }$. The same happens for fluctuations in the content with locally raised concentration, below the temperature $T_{\min }$. For $T_{\mathrm{dw}}<T<$ $T_{\min }$, regions with $\Delta^{(\text {of })}(x)<0$ partially suppress the magnetic order, although the bulk system remains globally in the FM phase. However, at the temperature $T_{\mathrm{dw}}(\Omega)$ the suppression becomes complete within a droplet; i.e., the order parameter vanishes at the origin, $\langle S(0)\rangle \sim \varphi_{\mathrm{dw}}(0)=0$. The state with kink-shaped magnetic order, $\langle S(x)\rangle \sim \varphi_{\mathrm{dw}}(x)$, covers the lowtemperature part of the phase diagram, $T<T_{\mathrm{dw}}$. By forming domain walls near the deep and wide hollows (the $\mathrm{Mn}_{\mathrm{d}}$ depleted regions) and therefore breaking the long-range order, the system prefers to change radically its magnetic structure in order to reduce the energy loss $\sim \int d x \Delta(x)\left[\varphi^{2}(x)-\bar{\varphi}^{2}\right]$. It is clear that the spatial regions containing such hollows in the concentration of $\mathrm{Mn}_{d}$ defects are very rare, and the complete violation of the long-range order occurs only in the one-dimensional model.

Of course, we are not able to describe adequately all the aspects of the unconventional behavior of such a complicated system as the $\mathrm{Si}: \mathrm{Mn}$ alloys with phase separation close to the Curie temperature. Nevertheless, the preceding analysis, carried out within the simplest version of the optimal fluctuation theory, shows that the $\mathrm{MnSi}_{2-z}$ precipitate with strong quenched disorder in the content of the $\mathrm{Mn}_{\mathrm{d}}$ defects could display very unusual FM states. These states are not obtained by a series expansion in the disorder around a critical point. It seems a reasonable and solid result that the FM phase transition in the precipitate is actually smeared in the sense that, within a wide temperature region, FM droplets coexist with the paramagnetic phase; in turn deformations are present against the uniform background of the phase with long-range FM order. Moreover magnetic structures with domain walls can appear. It should be noted that the aforementioned approach relies upon the assumption that the density of regions enriched or depleted in defects is sufficiently low to neglect their direct interplay.

\section{BOUNDARY-INDUCED FM ORDERING IN THE PRECIPITATE}

As we have previously discussed, the Si:Mn alloys exhibit a remarkable tendency toward phase segregation, when the dopant self-organizes into $\mathrm{MnSi}_{2-z}$ precipitate particles. These particles are surrounded by the insulating Si matrix in which the dopant is almost absent. Above, in Secs. III and IV, we have developed a model for magnetic ordering in the bulk $\mathrm{MnSi}_{2-z}$ precipitate. In the case of a $\mathrm{MnSi}_{2-z}$ precipitate particle, the previously stated results can obviously be affected by boundary effects in a finite-size system. For instance, the boundary between the precipitate and the matrix may strongly influence the magnetic characteristics of an individual precipitate nanoparticle. ${ }^{17,20}$

Below, we show that the spin-fluctuation scenario for high-temperature ferromagnetism in the finite-size precipitate embedded in the Si matrix is to some extent different from that of the bulk precipitate. We assume here that the interior of a precipitate particle consists of the pure $\mathrm{MnSi}_{1.7}$ silicide without $\mathbf{M n}_{\mathrm{d}}$ defects. The magnetic moments $\mathbf{S}_{i}$ are associated with unbound $3 d$ orbitals of $\mathrm{Mn}$ at the $\mathrm{MnSi}_{1.7} / \mathrm{Si}$ interface. The exchange coupling among the resulting interface magnetic moments is mediated by spin fluctuations, according to the 
mechanism described in Sec. III, and is therefore described by the effective Hamiltonian (10) with exchange integral (11), where the sites $i$ and $j$ belong to the boundary shell $b$ of a precipitate particle (henceforth, simply boundary).

We apply the mean-field approximation where each local moment at the boundary is influenced by the effective magnetic field $\left.\varphi(\mathbf{r})\right|_{b}$ produced by thermal spin fluctuations at the boundary $b$. On the other hand, the boundary magnetization of the local moments influences the carrier spin polarization inside the precipitate particle. The magnetic order is regarded to be essentially unaffected by disorder in the distribution of the local moments $\mathbf{S}_{i}$ at the boundary. Therefore, it is convenient to introduce a proper average of the spin density $\langle\varphi(\mathbf{r})\rangle$, which is uniform in the direction parallel to the interface but depends on the normal coordinate. The averaging procedure must be specified in each particular case. We connect the average magnetization of the local moments and spin density at the boundary through the relation

$$
\langle S\rangle=-S \mathcal{L}\left(\frac{\kappa S\left\langle\left.\varphi(\mathbf{r})\right|_{b}\right\rangle}{k_{B} T}\right) .
$$

Our forthcoming analysis is limited for simplicity to the case of collinear ordering and we indicate with $\mathbf{e}$ the unit vector along the magnetization direction, so that $\langle\varphi(\mathbf{r})\rangle=\mathbf{e}\langle\varphi(\mathbf{r})\rangle$ and $\langle\mathbf{S}\rangle=\mathbf{e}\langle S\rangle$. We shall show that a state with nonvanishing $\langle\varphi(\mathbf{r})\rangle$ and $\langle S\rangle$ occurs in the precipitate particle above the Curie temperature of the bulk of the homogeneous silicide, $T_{c}^{h}$, Eq. (3).

We choose to investigate three spatial configurations of precipitate: $\mathrm{MnSi}_{1.7}$ layer sandwiched between $\mathrm{Si}$ slabs and cylindrical and spherical precipitate particles inserted in the Si matrix (see Fig. 3). Such a choice is not incidental. Round-shaped precipitate nanoparticles are usually met in $\mathrm{Si}: \mathrm{Mn}$ alloys. Moreover, there is experimental evidence for the formation of vertical Mn-enriched nanopipes in $\mathrm{Si}_{1-x} \mathrm{Mn}_{x}$ alloys grown on a $\mathrm{Si}(100)$ substrate. ${ }^{27}$ Also the inspection of the local environment of $\mathrm{Mn}$ in the $\mathrm{Mn} \delta$-doped Si samples prepared by $\mathrm{MBE}$ on $\mathrm{Si}(001)$ substrates did not exclude a precipitation. ${ }^{28}$ We point out that, in the case of the layer, the averaging procedure to determine $\langle\varphi(\mathbf{r})\rangle$ consists in integrating over the Cartesian coordinates parallel to the layer (say, $y$ and $z$ ). The resulting average value still depend on the normal coordinate $x$. In the case of a cylindrical precipitate, the averaging procedure consists in integrating with respect to the angular variable $\phi$ and the axial coordinate $z$ in a suitable system of cylindrical coordinates. The resulting average still depends on the radial coordinate $\rho$. Finally, in the case of the spherical particle, the average is obtained integrating over the solid angle and still depends on the radial coordinate $r$.

To proceed with our analysis, we need to solve Eq. (6) for the function $\langle\varphi(\mathbf{r})\rangle$, in the right-hand side of which the potential $\lambda$ now reads

$$
\lambda(\mathbf{r})=\mathbf{e} \kappa\langle S\rangle \sigma_{m} f(\mathbf{r}),
$$

where the value of $\langle S\rangle$ is given by Eq. (32), $\sigma_{m}$ is the density of local magnetic moments, i.e., the number of local magnetic moments $\mathbf{S}_{i}$ per unit area of the boundary surface of the corresponding geometry (layer, cylinder, sphere). The

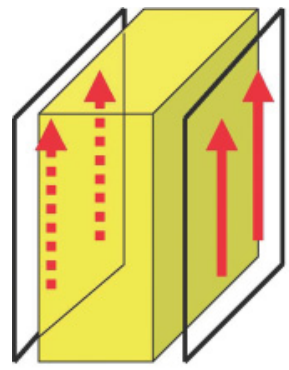

(a)

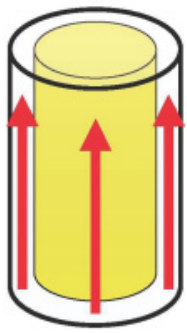

(b)

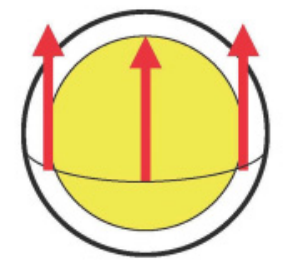

(c)

FIG. 3. (Color online) Sketch of the three spatial configurations of precipitate analyzed in our paper: (a) $\mathrm{MnSi}_{1.7}$ layer sandwiched between Si slabs, (b) cylindrical precipitate particle inserted in the $\mathrm{Si}$ matrix, and (c) spherical precipitate particle inserted in the Si matrix. The magnetic defects are assumed to be distributed on the surface of the precipitate particle, with surface density $\sigma_{m}$. Their magnetization is indicated by the arrows. The core part of the precipitate particle is indicated by the shaded region.

geometrical factor $f(\mathbf{r})$ has the following forms: $f(\mathbf{r})=\delta(x-$ $l)+\delta(x+l)$ for a layer of thickness $2 l ; f(\mathbf{r})=\delta\left(\rho-\rho_{0}\right)$, with $\rho=(x, y)$, for a cylinder of radius $\rho_{0}$ with the axis parallel to the $z$ axis; and $f(\mathbf{r})=\delta\left(r-r_{0}\right)$ for a sphere of radius $r_{0}$. Of course, outside the precipitate, in the Si matrix, the order parameter vanishes, $\langle\varphi(\mathbf{r})\rangle=0$.

In the case where the precipitate particle forms a layered configuration of the precipitate, the problem allows for an exact solution (see, e.g., Ref. 29). The spin density profile within the layer, $|x|<l$, is given by

$$
\langle\varphi(\mathbf{r})\rangle=\sqrt{\frac{\gamma}{\beta}} \frac{k^{\prime}}{\zeta_{k}} \mathrm{nc}\left(\frac{x}{\zeta_{k}}, k\right)
$$

where $k$ is the modulus of the elliptic function, $k^{\prime}=\sqrt{1-k^{2}}$, $\zeta_{k}=\zeta \sqrt{2 k^{2}-1}, 1>k^{2}>1 / 2, l / \zeta_{k}<K(k), K(k)$ is the complete elliptic integral of the first kind, and $\zeta$ is the correlation length of spin density fluctuations. Here, we are employing the standard notations for the elliptic functions and integrals. ${ }^{30}$ The quantity $k$ is determined by the boundary conditions at the interface via the equation

$$
\left.\gamma \frac{d\langle\varphi(\mathbf{r})\rangle}{d x}\right|_{x=l}=\frac{\kappa S \sigma_{m}}{2} \mathcal{L}\left(\frac{\kappa S\left\langle\left.\varphi(\mathbf{r})\right|_{x=l}\right\rangle}{k_{B} T}\right) .
$$

Taking the limit $k \rightarrow 1\left(k^{\prime} \rightarrow 0\right)$ in Eqs. (33) and (34), we obtain for the spin density profile near the transition 
temperature, $T \lesssim T_{c}^{l}$, the expressions

$$
\begin{gathered}
\langle\varphi(\mathbf{r})\rangle=\sqrt{\frac{\gamma}{\beta} \frac{k^{\prime}}{\zeta} \cosh \left(\frac{x}{\zeta}\right)} \\
\left(k^{\prime}\right)^{2}=\left[\frac{\zeta}{L} \operatorname{coth}\left(\frac{l}{\zeta}\right)-1\right]\left[g\left(\frac{l}{\zeta}\right)\right]^{-1}, \\
g(v)=1+\frac{1}{4} \cosh (2 \nu)+\frac{3 v}{2 \sinh (2 \nu)}+B \cosh ^{2}(v), \\
B=\frac{2}{15} \frac{\gamma}{\beta \zeta^{2}}\left(\frac{\kappa S}{k_{B} T}\right)^{2}, \\
L(T)=\frac{6 \gamma k_{B} T}{\kappa^{2} S^{2} \sigma_{m}} .
\end{gathered}
$$

Here, the so-called interpolation length $L$ is introduced. The equation $k^{\prime}=0$, together with Eq. (35), determines the condition under which the spin fluctuations freeze and the layer acquires a nontrivial magnetization $\langle S\rangle=$ $-\kappa S^{2}\left\langle\left.\varphi(\mathbf{r})\right|_{x=l}\right\rangle /\left(3 k_{B} T_{c}^{l}\right)$, i.e., the Curie temperature as a function the layer thickness and the defect density at the interface, $T_{c}^{l}\left(l, \sigma_{m}\right)$. As $l \gg L \simeq \zeta$, taking into account the temperature behavior of the correlation length $\zeta(T)$, Eq. (4), in the region $T \gg T_{c}^{h}$, we obtain the temperature of FM ordering at a single interface,

$$
T_{c}^{\infty}=\left[\frac{T_{0} \sigma_{m}^{2}(\kappa S)^{4}}{\left(6 \gamma k_{B} Q\right)^{2}}\right]^{1 / 3} .
$$

Although our theory cannot be pushed to the limit of too small precipitate particles, to illustrate the tendency of the critical temperature to increase with decreasing precipitate particle size, we can formally explore the region of parameters $l \ll \zeta<L$, where the temperature of the FM ordering in the $\mathrm{MnSi}_{1.7}$ layer may grow as high as

$$
T_{c}^{l}=\frac{\kappa S}{Q} \sqrt{\frac{T_{0} \sigma_{m}}{6 \gamma k_{B} l}} .
$$

Of course, this value must be considered as some estimate, obtained pushing the theory beyond its formal limit of validity.

Now, let us image that a $\mathrm{MnSi}_{1.7}$ cylinder of radius $\rho_{0}$ (infinite along the $z$ axis) is inserted in the Si matrix. In the linear approximation, the spin density profile on the $(x, y)$ plane has the form

$$
\begin{gathered}
\langle\varphi(\mathbf{r})\rangle=\sqrt{\frac{\gamma}{\beta}} \frac{p}{\zeta} I_{0}\left(\frac{\rho}{\zeta}\right), \\
p^{2}=\left[\frac{\zeta}{L} \frac{I_{0}\left(\rho_{0} / \zeta\right)}{I_{1}\left(\rho_{0} / \zeta\right)}-1\right]\left[g_{c}\left(\frac{\rho_{0}}{\zeta}\right)\right]^{-1}, \\
g_{c}(v)=\frac{2}{v I_{0}(v) I_{1}(v)} \int_{0}^{v} d t t I_{0}^{4}(t)+B I_{0}^{2}(v),
\end{gathered}
$$

where $I_{0}(v)$ and $I_{1}(v)$ are the modified Bessel functions of zeroth and first order, respectively. The condition $p=0$ in Eq. (38) determines the Curie temperature as a function of the cylinder radius and the defect density at the boundary, $T_{c}^{c}\left(\rho_{0}, \sigma_{m}\right)$. As $\rho_{0} \gg L \simeq \zeta$, the temperature of FM ordering at the boundary evidently equals $T_{c}^{\infty}$, Eq. (36). With the same words of caution as in the case of the layer precipitate particle, we can formally explore the case $\rho_{0} \ll \zeta<L$, which falls beyond the limit of validity of our approach, simply to illustrate the tendency of the critical temperature to increase with decreasing precipitate particle size. In the case of a $\mathrm{MnSi}_{1.7}$ cylinder (formally, in this limiting case, a filament), the critical temperature can grow as high as

$$
T_{c}^{c}=\frac{\kappa S}{Q} \sqrt{\frac{T_{0} \sigma_{m}}{3 \gamma k_{B} \rho_{0}}} .
$$

The specific magnetization (per unit length) of the cylindrical surface at $T \lesssim T_{c}^{c}$ is proportional to the value of the spin density, Eq. (37), at the boundary, $2 \pi \rho_{0} \sigma_{m} \kappa S^{2}\left\langle\left.\varphi(\mathbf{r})\right|_{\rho=\rho_{0}}\right\rangle /\left(3 k_{B} T_{c}^{c}\right)$.

Finally, let us imagine a $\mathrm{MnSi}_{1.7}$ sphere of radius $r_{0}$ inserted in the Si matrix. In the linear approximation, the spin density profile as a function of the radial coordinate $r$ has the form

$$
\begin{gathered}
\langle\varphi(\mathbf{r})\rangle=\sqrt{\frac{\gamma}{\beta}} \frac{q}{r} \sinh \left(\frac{r}{\zeta}\right), \\
q^{2}=\left\{\frac{\zeta}{L}\left[\operatorname{coth}\left(\frac{r_{0}}{\zeta}\right)-\frac{\zeta}{r_{0}}\right]^{-1}-1\right\}\left[g_{s}\left(\frac{r_{0}}{\zeta}\right)\right]^{-1}, \\
g_{s}(v)=\frac{\operatorname{shi}(4 v)-2 \operatorname{shi}(2 v)-2 \sinh ^{4}(v) / v}{\sinh (v)[\cosh (v)-\sinh (v) / v]}+B\left[\frac{\sinh (v)}{v}\right]^{2}, \\
\operatorname{shi}(v)=\int_{0}^{v} d t \frac{\sinh t}{t} .
\end{gathered}
$$

The condition $q=0$ in Eq. (40) determines the Curie temperature as a function the sphere radius and the defect density at the boundary, $T_{c}^{s}\left(r_{0}, \sigma_{m}\right)$. As $r_{0} \gg L \simeq \zeta$, the temperature of FM ordering at the boundary evidently equals $T_{c}^{\infty}$, Eq. (36). The magnetization of the sphere particle at $T \lesssim T_{c}^{s}$ is equal to $4 \pi r_{0}^{2} \sigma_{m} \kappa S^{2}\left\langle\left.\varphi(\mathbf{r})\right|_{r=r_{0}}\right\rangle /\left(3 k_{B} T_{c}^{s}\right)$, where, as usual, $\left.\langle\varphi(\mathbf{r})\rangle\right|_{r=r_{0}}$ is the value of the spin density, Eq. (39), at the boundary. As $r_{0} \gg L \simeq \zeta$, Eqs. (39) and (40) yield

$$
\left.\langle\varphi(\mathbf{r})\rangle\right|_{r=r_{0}}=Q \sqrt{\frac{3 \gamma}{\beta(1+2 B)} \frac{T_{c}^{\infty}-T}{T_{0}}},
$$

where $T_{c}^{\infty}$ is the temperature of the FM ordering near the interface which is evidently coincident with Eq. (36). Again, to illustrate the general trend, we can push the theory beyond its formal limit of validity and explore the limit of a relatively small sphere particle, $r_{0} \ll \zeta<L$. In this case the value of the order parameter at the interface is

$$
\left\langle\varphi\left(\mathbf{r}_{0}\right)\right\rangle=Q \sqrt{\frac{2 \gamma}{\beta(2+B)} \frac{T_{c}^{s}-T}{T_{0}}},
$$

where the transition temperature $T_{c}^{s}$ may formally become very large,

$$
T_{c}^{s}=\frac{\kappa S}{Q} \sqrt{\frac{T_{0} \sigma_{m}}{2 \gamma k_{B} r_{0}}} .
$$

A remark about the magnetic structure of the real $\mathrm{MnSi}_{1.7}$ silicide is now in order. In Ref. 11, below the transition temperature of about $40 \mathrm{~K}$, the magnetic order in bulk $\mathrm{Mn}_{4} \mathrm{Si}_{7}$ was identified as a spiral spin structure with a periodicity $\tau$ larger than $100 \AA$. Under the condition that such a spin structure persists in the case of restricted precipitate (the size 
of which is not much smaller than $\tau$ ), complicated noncollinear configuration of the interface moments could be realized due to the interplay in Eq. (32). In order to describe this phenomenon in detail, we should introduce additional relevant terms (in the form of Lifshitz invariants) into the initial free energy functional. However the main result of our previous analysis would not be significantly affected.

Of course, in the case of sufficiently large precipitate particles, it is understood that the mechanism of FM ordering in the finite-size precipitate due to the $\mathrm{MnSi}_{1.7} / \mathrm{Si}$ interface coexists with the spin-fluctuation mechanism discussed in Sec. III for the bulk precipitate.

\section{SUMMARY AND CONCLUDING REMARKS}

Our theoretical findings provide a general picture for understanding numerous intriguing magnetic properties observed in Si:Mn alloys. We presented these alloys as composed of $\mathrm{MnSi}_{2-z}$ precipitate particles enclosed in the Si matrix. ${ }^{7,10,17,20}$ We gave a detailed answer to the crucial question why the magnetization and the Curie temperature of the $\mathrm{MnSi}_{2-z}$ precipitate are much larger, roughly by an order of magnitude, than those of the bulk $\mathrm{MnSi}_{1.7}$ silicide (Ref. 11). We proceeded from the assumption that the $\mathrm{MnSi}_{2-z}$ precipitate is the $\mathrm{MnSi}_{1.7}$ silicide containing $\mathrm{Mn}_{\mathrm{d}}$ defects which carry local moments. These are coupled by an effective exchange via spin fluctuations in the $\mathrm{MnSi}_{1.7}$ weakly itinerant ferromagnetic host. Furthermore, local magnetic moments can be present not only inside the precipitate particle but also at the boundary between this particle and the silicon matrix. We showed that, for a precipitate particle, FM ordering may be augmented thanks to the enhanced concentration of local moments at the boundary. Under the assumption that quenched disorder in the defects' positions exists within the precipitate, the origin of specific features of high-temperature magnetism can be attributed to the short-range order in the form of FM droplets.

The available set of the experimental data supports directly or indirectly our assumptions and results. For instance, to decode the hysteresis curves of magnetization (of a rather complicated shape), the authors of Refs. 17 and 20 proposed the existence of two kinds of ferromagnets with different coercivities, which were ascribed to the coexistence of interface and inner magnetic moments in the precipitate particle. These explanations do not contrast with our scenario. In Ref. 14, where metallic $\mathrm{Si}_{1-x} \mathrm{Mn}_{x}$ films with high Mn content, $x \approx$ 0.35 , were studied, an anomalous Hall effect, with a hysteresis loop up to $T \approx 230 \mathrm{~K}$, was observed. It is reasonable to associate this phenomenon with the spin-fluctuation-mediated magnetism in bulk $\mathrm{MnSi}_{2-z}$ precipitate, but not with that of the $\mathrm{MnSi}_{1.7} / \mathrm{Si}$ boundary. The sharp decay of the resistivity detected below $T \approx 40 \mathrm{~K}$ provides evidence for the freezing of spin fluctuations in the samples. ${ }^{14}$ Moreover, a correlation between the concentration of magnetic scattering centers and the low-temperature resistivity as well the coercivity field is found in the experiments.

As we have previously discussed, the magnetic properties of the $\mathrm{MnSi}_{2-z}$ precipitate particle are significantly affected both by dimension effects and by the peculiarities and geometry of the $\mathrm{MnSi}_{1.7} / \mathrm{Si}$ boundary. For example, in the experiments of Refs. 7 and 10, on alloys with relatively low Mn content, the average diameter of a $\mathrm{MnSi}_{2-z}$ precipitate particle is $D_{0} \sim$ $11 \mathrm{~nm}$, each particle contains about $21000 \mathrm{Mn}$ ions and has a magnetic moment of $\sim 4300 \mu_{B}$. This means that, while the conditions for the validity of the point defect approximation, $D_{0} \gg a_{0} \gg d_{0}$, are generically satisfied, the continuous description of FM ordering in terms of a second-order phase transition is only qualitative, even without considering the effect of the boundary magnetization. However, even if at low temperatures, $T \approx T_{c}^{h}$, the condition $D_{0} \gg \zeta(T)$ is obviously not satisfied, at high temperatures, $T \approx T_{c}^{g}$, we have $\zeta\left(T_{c}^{g}\right) / D_{0}=$ $\left(Q D_{0}\right)^{-1} \sqrt{T_{0} / T_{c}^{g}} \propto(3-4)\left(k_{F} D_{0}\right)^{-1} \ll 1$. Thus, far above the temperature $T_{c}^{h}$ of intrinsic FM ordering in the host, the continuous approximation is formally valid.

In general, the fabrication of good epitaxial quality materials with higher $T_{c}$ using larger content of magnetic elements is accompanied by strong concentration fluctuations and even by the instability against phase separation. The formation of Mn-rich phases and their influence on the magnetic properties of the system is the key issue for magnetic materials based on group-IV semiconductors. Their behavior cannot be explained in the framework of standard models adopted in traditional DMSs and based on a mean-field theory. ${ }^{31}$ $A b$ initio calculations for such inhomogeneous disordered systems are extremely difficult and no method has been as yet established. In Ref. 32 the spinodal decomposition in $(\mathrm{Ga}, \mathrm{Mn}) \mathrm{As}$ and $(\mathrm{Ga}, \mathrm{Mn}) \mathrm{N}$ was simulated relying on the Monte Carlo method; the Curie temperatures were estimated by the random phase approximation with disorder taken into account. In this work, we adopt a simple but physically clear phenomenological model which relates the origin of hightemperature ferromagnetism with the precipitation observed in $\mathrm{Si}_{1-x} \mathrm{Mn}_{x}$ alloys.

Among magnetic materials based on group-IV semiconductors, Ge:Mn occupies a prominent role. The electronic structure of $\mathrm{Si}$ and $\mathrm{Ge}$ are similar; in thermal equilibrium, both magnetic alloys - Si:Mn and Ge:Mn—exhibit phase separation with the appearance of intermetallic compounds as a secondary phase. The following issue should be addressed: what is the main difference between the origin of hightemperature magnetism in the alloys (from the point of view of our approach)? The magnetic properties of the $\mathrm{Ge}_{1-x} \mathrm{Mn}_{x}$ samples, with $x \gtrsim 1-3$ at. \% (for instance, the hysteretical loops of magnetization versus field up to room temperatures), are mainly associated with the presence of the Mn-rich inclusions in the form of $\mathrm{Mn}_{5} \mathrm{Ge}_{3}$ nanoprecipitates dispersed in a defectfree Ge crystal matrix. ${ }^{33,34}$ The film samples fabricated by low-temperature molecular beam epitaxy at $T_{\text {substrate }}>70{ }^{\circ} \mathrm{C}$ is characterized by a magnetization versus temperature curve shape similar to the one observed for the bulk $\mathrm{Mn}_{5} \mathrm{Ge}_{3}$ germanide; the saturation magnetization of the nanoparticles (per $\mathrm{Mn}$ atom) is only slightly smaller than that of bulk $\mathrm{Mn}_{5} \mathrm{Ge}_{3}$. ${ }^{35}$ The $\mathrm{Mn}_{5} \mathrm{Ge}_{3}$ compound is known to be a strong ferromagnet with a Curie temperature of $296 \mathrm{~K}$ and with large spin polarization, $2.74 \mu_{B} / \mathrm{Mn}^{36-38}$ Therefore, in the $\mathrm{Ge}_{1-x} \mathrm{Mn}_{x}$ alloys, the magnetization onset near room temperature is interpreted as the paramagnet to ferromagnet transition of the individual $\mathrm{Mn}_{5} \mathrm{Ge}_{3}$ precipitates. In the phasesegregated $\mathrm{Ga}_{1-x} \mathrm{Mn}_{x}$ As layers (where $x \simeq 4.7 \%-5.5 \%$ ), ferromagnetism observed up to a Curie temperature of $318 \mathrm{~K}$ 
can in an analogous manner be ascribed to the presence of MnAs magnetic precipitates within the GaAs matrix. ${ }^{39,40}$

In contrast to the above scenario, bulk $\mathrm{MnSi}_{1.7}$ silicide shows weak itinerant magnetism with an ordering temperature of $47 \mathrm{~K}$ and with a very low saturation moment of $0.012 \mu_{B} / \mathrm{Mn},{ }^{11}$ which are much different from those of the $\mathrm{MnSi}_{2-z}$ nanoparticles in the $\mathrm{Si}_{1-x} \mathrm{Mn}_{x}$ materials. ${ }^{10,17}$ The mechanism for high-temperature FM ordering in the $\mathrm{MnSi}_{2-z}$ precipitate due to spin fluctuations is presented in our work. Note that the saturation moment for the $\mathrm{MnSi}_{2-z}$ precipitate $\left(\simeq 0.2 \mu_{B} / \mathrm{Mn}\right)$ is much smaller than that for $\mathrm{Mn}_{5} \mathrm{Ge}_{3}$.

In this work, we did not address the problem of the exchange interaction among the $\mathrm{MnSi}_{2-z}$ precipitate particles. However it would be worth spending a few words on this issue. An ensemble of nanomagnets embedded into the matrix exhibits rich magnetic properties which are connected to the electron (transport) properties of the matrix. Preliminary estimates show that the precipitate particles could interact with one another through the insulating matrix that contains defects with shallow impurity levels of depth $\epsilon_{f} \ll\left(W, E_{g}\right)$ inside the gap $E_{g}$. The relevant effective coupling is likely to be of the FM type with an exponential decay on the scale $\propto\left(\sqrt{2 m \epsilon_{f}}\right)^{-1} \propto a \sqrt{W / \epsilon_{f}}$, which may considerably exceed interatomic distances $a$. The existence of defect localized states of carriers (holes) inside the gap depends on the particular combination of matrix crystal and impurity atom, on the position of the latter within the lattice (substitution or interstitial), and on the surrounding environment. We recall a number of interesting articles, Refs. 41-44, dedicated to this important topic. As far as an ensemble of nanomagnets is concerned, at sufficiently high temperatures (but, obviously, below the temperature of FM transition inside the precipitate) the superparamagnetic behavior of the particles is almost evident,${ }^{10}$ but in the low-temperature region, the FM alignment of magnetic moments of different particles is possible. On the other hand, the blocking of these moments by the matrix and the formation of a cluster-glass state may not be excluded.

\section{ACKNOWLEDGMENTS}

The work was partially supported by the University of the Basque Country (Proyecto GV UPV/EHU Grant No. IT-366-07), the Spanish Ministerio de Ciencia y Tecnologia (Grant No. FIS2007-66711-C02-01), and the RFBR (Grant No. 10-02-00118). SC also acknowledges financial support by PRIN 2007 under Project No. 2007FW3MJX003. VVT acknowledges financial support by Ikerbasque (Basque Foundation for Science).
${ }^{1}$ F. M. Zhang, X. C. Liu, J. Gao, X. S. Wu, Y. W. Du, H. Zhu, Q. Xiao, and P. Chen, Appl. Phys. Lett. 85, 786 (2004).

${ }^{2}$ M. Bolduc, C. Awo-Affouda, A. Stollenwerk, M. B. Huang, F. G. Ramos, G. Agnello, and V. P. LaBella, Phys. Rev. B 71, 033302 (2005).

${ }^{3}$ X. C. Liu, Z. H. Lu, Z. L. Lu, L. Y. Lv, X. S. Wu, F. M. Zhang, and Y. W. Du, J. Appl. Phys. 100, 073903 (2006); X. C. Liu, Y. B. Lin, J. F. Wang, Z. H. Lu, Z. L. Lu, J. P. Xu, L. Y. Lv, F. M. Zhang, and Y. W. Du, ibid. 102, 033902 (2007).

${ }^{4}$ S. H. Chiu, H. S. Hsu, and J. C. A. Huang, J. Appl. Phys. 103, 07D110 (2008).

${ }^{5}$ A. Stroppa, S. Picozzi, A. Continenza, and A. J. Freeman, Phys. Rev. B 68, 155203 (2003).

${ }^{6}$ A. Wolska, K. Lawniczak-Jablonska, M. Klepka, M. S. Walczak, and A. Misiuk, Phys. Rev. B 75, 113201 (2007).

${ }^{7}$ S. Zhou, K. Potzger, G. Zhang, A. Mücklich, F. Eichhorn, N. Schell, R. Grötzschel, B. Schmidt, W. Skorupa, M. Helm, J. Fassbender, and D. Geiger, Phys. Rev. B 75, 085203 (2007).

${ }^{8}$ L. Zeng, E. Helgren, M. Rahimi, F. Hellman, R. Islam, B. J. Wilkens, R. J. Culbertson, and D. J. Smith, Phys. Rev. B 77, 073306 (2008). ${ }^{9}$ C. Awo-Affouda, M. Bolduc, M. B. Huang, F. G. Ramos, K. A. Dunn, B. Thiel, G. Agnello, and V. P. LaBella, J. Vac. Sci. Technol. A 24, 1644 (2006).

${ }^{10}$ S. Zhou, A. Shalimov, K. Potzger, M. Helm, J. Fassbender, and H. Schmidt, Phys. Rev. B 80, 174423 (2009).

${ }^{11}$ U. Gottlieb, A. Sulpice, B. Lambert-Andron, and O. Laborde, Alloys Compd. 361, 13 (2003); A. Sulpice, U. Gottlieb, M. Affronte, and O. Laborde, J. Magn. Magn. Mater. 272-276, 519 (2004).

${ }^{12}$ M. Lee, Y. Onose, Y. Tokura, and N. P. Ong, Phys. Rev. B 75, 172403 (2007).
${ }^{13}$ V. Ko, K. L. Teo, T. Liew, T. C. Chong, M. MacKenzie, I. MacLaren, and J. N. Chapman, J. Appl. Phys. 104, 033912 (2008).

${ }^{14}$ S. N. Nikolaev, B. A. Aronzon, V. V. Ryl'kov, V. V. Tugushev, E. S. Demidov, S. A. Levchuk, V. P. Lesnikov, V. V. Podol'skii, and R. R. Gareev, JETP Lett. 89, 603 (2009).

${ }^{15}$ A. F. Orlov, A. B. Granovsky, L. A. Balagurov, I. V. Kulemanov, Yu. N. Parkhomenko, N. S. Perov, E. A. Gan'shina, V. T. Bublik, K. D. Shcherbachev, A. V. Kartavykh, V. I. Vdovin, A. Sapelkin, V. V. Saraikin, Yu. A. Agafonov, V. I. Zinenko, A. Rogalev, and A. Smekhova, J. Exp. Theor. Phys. 109, 602 (2009).

${ }^{16}$ S. V. Adashkevich, N. M. Lapchuk, V. F. Stel'makh, G. G. Fedoruk, and E. N. Shumskaya, JETP Lett. 84, 547 (2006).

${ }^{17}$ S. Yabuuchi, Y. Ono, M. Nagase, H. Kageshima, A. Fujiwara, and E. Ohta, Jpn. J. Appl. Phys. 47, 4487 (2008).

${ }^{18}$ T. Jungwirth, K. Y. Wang, J. Masek, K. W. Edmonds, J. König, J. Sinova, M. Polini, N. A. Goncharuk, A. H. MacDonald, M. Sawicki, A. W. Rushforth, R. P. Campion, L. X. Zhao, C. T. Foxon, and B. L. Gallagher, Phys. Rev. B 72, 165204 (2005).

${ }^{19}$ D. B. Migas, V. L. Shaposhnikov, A. B. Filonov, V. E. Borisenko, and N. N. Dorozhkin, Phys. Rev. B 77, 075205 (2008).

${ }^{20}$ S. Yabuuchi, H. Kageshima, Y. Ono, M. Nagase, A. Fujiwara, and E. Ohta, Phys. Rev. B 78, 045307 (2008).

${ }^{21}$ Q. Liu, W. Yan, H. Wei, Z. Sun, Z. Pan, A. V. Soldatov, C. Mai, C. Pei, X. Zhang, Y. Jiang, and S. Wei, Phys. Rev. B 77, 245211 (2008).

${ }^{22}$ V. N. Men'shov, V. V. Tugushev, and S. Caprara, Eur. Phys. J. B 77, 337 (2010)

${ }^{23}$ T. Moriya, Spin Fluctuation in Itinerant Electron Magnetism (Springer, Berlin, 1985).

${ }^{24}$ K. K. Murata and S. Doniach, Phys. Rev. Lett. 29, 285 (1972). 
${ }^{25}$ A. P. Levanyuk, V. V. Osipov, A. S. Sigov, and A. A. Sobyanin, Sov. Phys. JETP 49, 176 (1979).

${ }^{26}$ L. N. Bulaevskii, S. V. Panyukov, and M. V. Sadovskii, Sov. Phys. JETP 65, 380 (1987).

${ }^{27}$ Y. Zhang, Q. Jiang, D. J. Smith, and J. Drucker, J. Appl. Phys. 98, 033512 (2005).

${ }^{28}$ Q. F. Xiao, S. Kahwaji, T. L. Monchesky, R. A. Gordon, and E. D. Crozier, J. Phys. Conf. Series 190, 012101 (2009).

${ }^{29}$ O. Navarro, V. N. Men'shov, and V. V. Tugushev, Phys. Rev. B 72, 224430 (2005).

${ }^{30}$ Handbook of Mathematical Functions, edited by M. Abramowitz and I. A. Stegun (Dover, New York, 1971).

${ }^{31}$ T. Dietl, H. Ohno, and F. Matsukura, Phys. Rev. B 63, 195205 (2001).

${ }^{32}$ K. Sato, H. Katayama-Yoshida, and P. H. Dederichs, Jpn. J. Appl. Phys. 44, L948 (2005).

${ }^{33}$ S. Ahlers, D. Bougeard, N. Sircar, G. Abstreiter, A. Trampert, M. Opel, and R. Gross, Phys. Rev. B 74, 214411 (2006).

${ }^{34}$ C. Bihler, C. Jaeger, T. Vallaitis, M. Gjukic, M. S. Brandt, E. Pippel, J. Woltersdorf, and U. Gösele, Appl. Phys. Lett. 88, 112506 (2006).
${ }^{35}$ P. De Padova, J.-P. Ayoub, I. Berbezier, P. Perfetti, C. Quaresima, A. M. Testa, D. Fiorani, B. Olivieri, J.-M. Mariot, A. Taleb Ibrahimi, M. C. Richter, O. Heckmann, and K. Hricovini, Phys. Rev. B 77, 045203 (2008).

${ }^{36}$ S. Picozzi, A. Continenza, and A. J. Freeman, Phys. Rev. B 70, 235205 (2004).

${ }^{37}$ C. Zeng, S. C. Erwin, L. C. Feldman, A. P. Li, R. Jin, Y. Song, J. R. Thompson, and H. H. Weitering, Appl. Phys. Lett. 83, 5002 (2003).

${ }^{38}$ J. B. Forsyth and P. J. Brown, J. Phys. Condens. Matter 2, 2713 (1990).

${ }^{39}$ I. T. Yoon, T. W. Kanga, and D. J. Kim, J. Magn. Magn. Mater. 320, 662 (2008).

${ }^{40}$ K. W. Edmonds, A. A. Freeman, N. R. S. Farley, K. Y. Wang, R. P. Campion, B. L. Gallagher, C. T. Foxon, G. van der Laan, and E. Arenholz, J. Appl. Phys. 102, 023902 (2007).

${ }^{41}$ A. Stroppa and G. Kresse, Phys. Rev. B 79, 201201(R) (2009).

${ }^{42}$ A. Stroppa, S. Picozzi, A. Continenza, M. Y. Kim, and A. J. Freeman, Phys. Rev. B 77, 035208 (2008).

${ }^{43}$ A. Stroppa, X. Duan, M. Peressi, D. Furlanetto, and S. Modesti, Phys. Rev. B 75, 195335 (2007).

${ }^{44}$ J. Mašek and F. Máca, Phys. Rev. B 69, 165212 (2004). 